Mittal and Lee et. al.

\title{
Targeting TRIP13 in Wilms Tumor with Nuclear Export Inhibitors
}

Karuna Mittal ${ }^{1,2^{*}}$, Benjamin P. Lee ${ }^{1,2^{*}}$, Garrett W. Cooper ${ }^{1,2}$, Jenny Shim ${ }^{1,2}$, Hunter C. Jonus ${ }^{1,2}$, Won Jun $\mathrm{Kim}^{3,4}$, Mihir Doshi ${ }^{3,4}$, Diego Almanza, ${ }^{3,4}$, Bryan D. Kynnap ${ }^{3,4}$, Amanda L. Christie ${ }^{3}$, Xiaoping Yang ${ }^{4}$, Glenn S. Cowley ${ }^{4}$, Brittaney A. Leeper ${ }^{5}$, Christopher L. Morton ${ }^{6}$, Bhakti Dwivedi ${ }^{7}$, Taylor Lawrence ${ }^{1,2}$, Manali Rupji ${ }^{8}$, Paula Keskula ${ }^{4}$, Stephanie Meyer ${ }^{9}$, Catherine M. Clinton ${ }^{9}$, Manoj Bhasin ${ }^{1,2,8}$, Brian D. Crompton $^{4,9}$, Yuen-Yi Tseng ${ }^{4}$, Jesse S. Boehm ${ }^{4}$, Keith L. Ligon ${ }^{4,10,11}$, David E. Root ${ }^{4}$, Andrew J. Murphy ${ }^{6}$, David M. Weinstock ${ }^{4,9}$, Prafulla C. Gokhale ${ }^{5}$, Jennifer M. Spangle ${ }^{7,8}$, Miguel N. Rivera ${ }^{4,12}$, Elizabeth A. Mullen $^{4}$, Kimberly Stegmaier ${ }^{4,9}$, Kelly C. Goldsmith ${ }^{1,2,8}$, William C. Hahn ${ }^{3,4^{* *}}$, Andrew L. Hong ${ }^{1,2,8^{* *}}$

${ }^{1}$ Department of Pediatrics, Emory University School of Medicine, Atlanta, GA

${ }^{2}$ Aflac Cancer and Blood Disorders Center, Children's Healthcare of Atlanta, Atlanta, GA

${ }^{3}$ Department of Medical Oncology, Dana-Farber Cancer Institute, Boston, MA, USA

${ }^{4}$ Broad Institute of MIT and Harvard, Cambridge, MA, USA

${ }^{5}$ Experimental Therapeutics Core and Belfer Center for Applied Cancer Science, Dana-Farber Cancer Institute, Boston, Massachusetts.

${ }^{6}$ Department of Surgery, St. Jude Children's Research Hospital, Memphis, Tennessee

${ }^{7}$ Department of Radiation Oncology, Emory University School of Medicine, Atlanta, GA

${ }^{8}$ Winship Cancer Institute, Emory University School of Medicine, Atlanta, GA

${ }^{9}$ Department of Pediatric Oncology, Dana-Farber Cancer Institute, Boston, MA, USA

${ }^{10}$ Department of Pathology, Brigham and Women's Hospital, Boston, MA, USA

${ }^{11}$ Department of Oncologic Pathology, Dana-Farber Cancer Institute, Boston, MA, USA

${ }^{12}$ Department of Pathology, Massachusetts General Hospital, Boston, MA, USA

${ }^{*}$ These authors contributed equally

**Co-senior authors and co-corresponding authors:

Andrew L. Hong, M.D.

Department of Pediatrics

Emory University

1760 Haygood Drive NE

Atlanta, GA 30322

404-727-3720 (phone)

41 andrew.hong2@emory.edu

William C. Hahn, M.D., Ph.D.

Department of Medical Oncology

Dana-Farber Cancer Institute

450 Brookline Avenue, Dana 1630

Boston, MA 02215

617-632-5244 (phone)

william_hahn@dfci.harvard.edu 
Mittal and Lee et. al.

\section{ABSTRACT}

44 Wilms tumor (WT) is the most common renal malignancy of childhood. Despite improvements in the 45 overall survival, relapse occurs in $\sim 15 \%$ of patients with favorable histology WT (FHWT). Half of these

46 patients will succumb to their disease. Identifying novel targeted therapies in a systematic manner remains

47 challenging in part due to the lack of faithful preclinical in vitro models. We established ten short-term

48 patient-derived WT cell lines and characterized these models using low-coverage whole genome

49 sequencing, whole exome sequencing and RNA-sequencing, which demonstrated that these ex-vivo models

50 faithfully recapitulate WT biology. We then performed targeted RNAi and CRISPR-Cas9 loss-of-function

51 screens and identified the nuclear export genes (XPO1 and KPNB1) as strong vulnerabilities. We observed

52 that these models are sensitive to nuclear export inhibition using the FDA approved therapeutic agent,

53 selinexor (KPT-330). Selinexor treatment of FHWT suppressed TRIP13 expression, which was required

54 for survival. We further identified in vitro and in vivo synergy between selinexor and doxorubicin, a

55 chemotherapy used in high risk FHWT. Taken together, we identified XPO1 inhibition with selinexor as a

56 potential therapeutic option to treat FHWTs and in combination with doxorubicin, leads to durable

57 remissions in vivo. 
Mittal and Lee et. al.

\section{INTRODUCTION}

59 Wilms tumor (WT) is the most common childhood renal tumor and represents $\sim 6 \%$ of all pediatric cancers

60 with a peak age of presentation at 3 years ${ }^{1-3}$. In the United States, African-American children have 2.5 times

61 higher rates of WT when compared to Caucasians or Asian-American children ${ }^{4-6}$. For low-risk disease,

62 current therapy includes the use of surgery and chemotherapy (e.g., vincristine, dactinomycin). For high-

63 risk disease (e.g. pulmonary metastasis or invasion of the renal sinus and capsule), doxorubicin and

64 radiation therapy are added to low-risk disease therapy. Despite significant increases in response and

65 survival over the past 50 years, $\sim 15 \%$ of patients with WT recur ${ }^{7-9}$ and salvage regimens are successful only

66 in 50\% of patients and carry significant morbidity.

A major limiting factor in testing novel targeted therapies in WT is the lack of faithful in vitro preclinical models. Prior cell line models of WT have been recharacterized as other pediatric cancers such as rhabdoid tumor (e.g. G401) and Ewing sarcoma (e.g. SK-NEP) ${ }^{10-13}$. Recent efforts to generate WT cell lines have been limited due to finite passaging ${ }^{14-17}$. Despite this limitation, a repository of WT organoids and patient derived xenografts (PDXs) has been developed in recent years ${ }^{18-20}$.

74 Nomination of rational therapeutics for organoid or in vivo PDX studies, however, requires systematic in

75 vitro efforts using faithful cancer cell lines. Here, we have developed faithful cell line models using genome 76 and transcriptome sequencing of WT which recapitulate known WT biology. We then performed functional 77 genomic screens focused on druggable targets to nominate WT therapeutics ${ }^{21-23}$.

\section{RESULTS}

\section{WT cell lines faithfully recapitulate genomic and transcriptomic features of WT}

82 Recent studies have shown the feasibility of generating short-term WT cell lines with limited genomic

83 testing ${ }^{14-17}$. We developed 10 short-term WT cell lines from 8 patients ( 8 patient derived cell lines and 2 
Mittal and Lee et. al.

PDX derived cell lines following one passage of the PDX in mice (Annotated as T2); Fig 1a; Methods).

Six patient samples were obtained at time of diagnosis and two were obtained at time of recurrence and one patient had diffuse anaplastic Wilms Tumor (DAWT; CCLF_PEDS_0023_T).

We then performed ultra-low coverage whole genome sequencing (WGS) to infer copy number status, whole exome sequencing (WES) to identify known mutations in WT and RNA-sequencing to assess the transcriptome in the patients' tumor and the matched cell lines. We identified 1q gain, a poor prognostic

92 factor in Wilms tumor biology, in one patient (CCLF_PEDS_0023_T) ${ }^{24,25}$. We observed gain in

93 chromosome 12 in two patients but did not identify LOH in $1 \mathrm{p}$ or $11 \mathrm{p} 15$. We then assessed the mutational

94 profiles of these tumors and cell lines (Fig 1a; Supp Table 1). We found that the mutations reflected the spectrum of mutations seen in the WT samples profiled in the NCI Therapeutically Applicable Research to Generate Effective Treatments ${ }^{26}$ (TARGET; Fig 1a). Finally, we identified TP53 (c.469G>T) in CCLF_PEDS_0023_T which is consistent with the pathological diagnosis of anaplastic Wilms tumor ${ }^{26}$ manifold approximation and projection (UMAP) ${ }^{27}$ and compared it to TARGET ${ }^{28}$ and St. Jude Children's

102 Research Hospital's WT datasets ${ }^{19}$ (Methods). We observed that our WT cell lines clustered closely with

103 the WT tumor samples (Fig 1b). We further observed that SIX2 and CITED1, genes involved in WT

104 biology, were upregulated in our WT cell lines consistent with WT biology (Fig 1c and d) $)^{29-31}$. Collectively, 105 our findings suggest that our patient-derived cell lines recapitulate known biology and serve as faithful 106 representations of WT. 
Mittal and Lee et. al.

We then asked if we could identify genetic vulnerabilities in WT despite the short-term lifespan of these

110 cell lines. Following rapid expansion of these cell lines within the first five passages from obtaining the

111 patient's sample, we subjected a subset of cell lines to targeted loss of function RNA interference and

112 CRISPR-Cas9 screens. Through these screens, we identified genes that lead to decreased cell viability when

113 suppressed or deleted. As cell numbers were limited, we used the Druggable Cancer Targets (DCT)

114 libraries, a library of 429 genes, as previously described ${ }^{23,32}$ (Fig 2a).

116 For the RNAi screens, we introduced the DCT lentiviral library into the CCLF_PEDS_0002_T,

117 CCLF_PEDS_0041_T1, and CCLF_PEDS_0041_T2 cells and compared the abundance of each shRNA at

118 the time of infection to its abundance after 25-29 days of cell culture (Supp Fig 1a-c). We then used Model-

119 based Analysis of Genome-wide CRISPR/Cas9 Knockout (MAGeCK) to identify 20 genes required for

120 survival across these cell lines ${ }^{33}$ (Fig 2b). We subsequently performed CRISPR-Cas9 screens in

121 CCLF_PEDS_0002_T, CCLF_PEDS_0023_T and CCLF_PEDS_0023_T2 to identify 24 genes that when

122 deleted led to decreased viability across these cell lines (Supp Fig 1d-f). From these orthogonal screens,

123 we identified eight genes which overlapped. These included genes involved in nuclear export

124 (KPNB1 and XPO1), regulators of the cell cycle (KIF11 and POLA1), DNA damage (UBA1 and DDB1)

125 and cell survival (BIRC5) (Fig 2b).

127 We focused on the role of nuclear export since inhibitors such as selinexor (KPT-330) have been developed

128 and recently FDA-approved in multiple myeloma and diffuse large B-cell lymphoma ${ }^{34}$. We first assessed

129 the expression levels of XPOI across 85 cancer types through the University of California Santa Cruz

130 Treehouse Childhood Cancer Initiative ${ }^{35}$. From 12,719 patient samples, we found $85.4 \%$ of Wilms Tumor

131 samples were in the top $20 \%$ of samples with high XPOI levels (Fig 2c). To validate the requirement of

132 XPO1 in WT cell lines, we assessed cell viability of WT cells using KPT-330 and XPO1 suppression with

133 RNAi (Fig 2d, Sup fig 2a-b). Specifically, we determined the $\mathrm{IC}_{50}$ values for our WT cell lines and 134 compared these to adjacent normal kidney cell lines (PEDS_0023_N, PEDS_066N, PEDS_040N). We 
Mittal and Lee et. al.

135 observed that cell lines from adjacent normal renal tissue were not sensitive to KPT-330 with an $\mathrm{IC}_{50}$

136 ranging 10-20 uM (Fig 2d, Supp fig 2c). In contrast, our WT cell lines had up to 10-fold lower $\mathrm{IC}_{50}$

137 consistent with responses seen in multiple myeloma where KPT-330 has FDA approval ${ }^{36}$. Specifically, half 138 of these cell lines showed an $\mathrm{IC}_{50}$ at nanomolar concentrations (e.g. 25-800nM) whereas the other cell lines

139 had an $\mathrm{IC}_{50}$ in the low micromolar range (e.g., 1-5 $\mu \mathrm{M}$ ). On-target activity of KPT-330 requires

140 transcriptional upregulation of $X P O 1$ due to an auto-feedback loop in conjunction with suppression of 141 XPO1 protein levels ${ }^{37}$. However the downstream mechanisms of action varies by cancer type. Consistent 142 with these prior findings, we saw upregulation of XPO1 by qRT-PCR and down regulation of XPO1 protein 143 in WT (Fig 2e and Supp Fig 2d). Taken together, these findings suggest that XPO1 is a potential 144 therapeutic target in WT.

XPO1 inhibition induces cell death through the TRIP13/ TP53 axis

147 We next investigated a potential mechanism of action of the nuclear export inhibitor KPT-330 in WT. KPT148330 is known to affect tumor suppressors such as a TP53 through nuclear accumulation of TP53 and 149 prevention of MDM2/4 related degradation ${ }^{38}$. We focused our efforts in understanding the mechanisms in 150 FHWT. We treated TP53 wild-type CCLF_PEDS_0041_T1 with DMSO or KPT-330 and performed RNA 151 sequencing (Methods). We then performed differential expression analyses and then examined gene sets 152 enriched or suppressed upon KPT-330 treatment using Gene Set Enrichment Analyses (GSEA) ${ }^{39}$. We found 153412 genes differentially expressed (Fig 3a) and 50 hallmark gene sets significantly enriched or suppressed 154 (Supp Table 3).

156 We found gene sets affecting both G1 (e.g. E2F) and G2/M pathways (Fig 3b) along with activation of the 157 TP53 pathway (Supp Table 2). Other processes such as apoptosis were also enriched. We performed 158 fluorescence-activated single cell analyses to assess the changes in the cell cycle following KPT-330 159 treatment. We found that changes in G1 were not consistent across our FHWT cell lines (Fig 3c). However, 160 we saw decreases of S phase and consistent increases in G2/M suggesting that KPT-330 in our FHWT cell 
Mittal and Lee et. al.

161 lines led primarily to a G2/M arrest (Fig 3c). We then confirmed the activation of TP53 through nuclear 162 accumulation of TP53 following treatment with KPT-330 by 48 hours (Fig 2e; Supp fig 3a). As KPT-330

163 has been associated with upregulating nuclear accumulation of TP53, we sought to determine if TP53 was 164 indeed an important mechanism within WT ${ }^{40}$. We depleted TP53 using CRISPR-Cas9 in PEDS1012T and 165 PEDS_0022_T as compared to a control gRNA to LacZ (Supp Fig 3b). We then assessed the IC 50 of KPT166330 and found a 6 to 13- fold increase in these values when TP53 was deleted (Fig 3d, Supp Fig 3c). This 167 significant change in $\mathrm{IC}_{50}$ s suggests a critical role of TP53 in KPT-330 induced cell death in FHWT.

169 We subsequently assessed the 412 differentially expressed genes and observed that the expression of 170 TRIP13, Thyroid Hormone Receptor Interactor 13, was significantly downregulated in the KPT-330 treated 171 cells (Fig 3a). TRIP13 previously was found to interact with co-factors of TP53 in injured renal epithelial 172 cells ${ }^{41}$. More recently, TRIP13 has been identified as a cancer predisposition gene in WT ${ }^{42}$.

174 We then focused on the function of TRIP13 in our FHWT cell lines. First, we observed that in general, 175 pediatric renal tumors had modest increases in expression of TRIP13 (Supp Fig 3d) as compared to adjacent 176 normal kidney controls. We then suppressed the expression of TRIP13 with RNAi in TP53 wild-type WT 177 cells using two different TRIP13 shRNA constructs (Methods). To mitigate off-target effects, we used a 178 seed control to one shRNA construct and an RFP non-targeting control ${ }^{43}$. Our cell viability results showed 179 significant cell death in our cell lines transduced with either TRIP13 shRNA constructs (14-59\% cell 180 viability; Fig 3e and Supp Fig 3e-f). In addition, when we overexpressed TRIP13 in our FHWT cell lines, 181 we found a modest $15-27 \%$ increase in cell counts as compared to a luciferase overexpression control (Supp 182 Fig 3g). We then tried to rescue our shTRIP13 viability defect using this overexpression vector but the 3' 183 UTR shRNA alone did not lead to a viability defect or suppression of TRIP13 by immunoblot (Supp Fig 184 3e; shTRIP13-3). However, in our TP53 deleted cells, we found that TRIP13 levels elevated at baseline 185 and did not decrease upon KPT-330 treatment (Supp Fig 3h). These results suggest that FHWT (e.g., TP53 
Mittal and Lee et. al.

186

wild type) cells require TRIP13 for survival and when overexpressed, lead to a modest increase in proliferation.

We then assessed the transcriptional changes by RNA sequencing seen when we suppress TRIP13. We used the CCLF_PEDS_0041_T1 and AFLAC_2377 cell lines to determine the consequences of suppressing TRIP13 as compared to our non-targeting shRNA controls (Methods). We found 172 genes upregulated and 101 genes downregulated (Fig 3f). We used GSEA to identify gene sets significantly enriched upon suppression of TRIP13. We found the gene sets involving RB1/RBL1 skin specific knockout mice as significant which was confirmed with decreased cyclin D1 levels (Fig 3f-g; Supp fig 3f) ${ }^{44}$. We further found, similar to XPO1 inhibition, that TRIP13 is anti-correlated with the G2/M checkpoint (Fig 3g).

We subsequently asked how suppression of TRIP13 contributed to the phenotypes seen when FHWT cells were treated with KPT-330. We looked at the overlap between differentially expressed genes from our RNA-sequencing experiment (Fig 3h). We found eight genes upregulated and four genes downregulated. Interestingly, upregulated genes $C D H 1, M Y C T 1$ and $S A M D 9$ have been implicated as tumor suppressors in other cancers such as hematologic malignancies ${ }^{45-47}$. When we evaluated the down-regulated genes with TRIP13 suppression, we find genes involved in the cell cycle such as $A S P M$ and $C D C A 7$. ASPM is essential for mitotic spindle function in neurons ${ }^{48}$ and CDCA7 regulates CCNA2, a cyclin with roles in G1 and $\mathrm{G} 2 / \mathrm{M}$, in esophageal squamous cell carcinomas ${ }^{49}$.

In sum, our findings show that treatment with KPT-330 in part leads to suppression of TRIP13 which in turn leads to alterations in the cell cycle.

\section{KPT-330 and doxorubicin are synergistic in vitro and in vivo in WT}

Doxorubicin was added to vincristine and dactinomycin in Stage III FHWT patients in the 1980s to account for higher risk disease (e.g., pulmonary metastasis or tumor rupture) ${ }^{50}$. Despite improved overall survival 
Mittal and Lee et. al.

212 with the addition of doxorubicin in patients with higher risk FHWT, these cancers recur in $15 \%$ of patients

$213^{8}$. Over the past two decades, only one Phase II clinical trial for relapsed FHWT has been opened

214 (clinicaltrials.gov: NCT04322318). This trial uses a chemotherapy backbone without novel therapeutic

215 targets. Thus, there is a need to identify potential synergistic combination strategies which could be tested

216 in the Phase I/II setting.

218 We assessed the role of adding KPT-330 to doxorubicin to determine if there was synergy with our cell line

219 models of FHWT. We first evaluated doxorubicin sensitivity in these patient-derived WT cell lines. We

220 found that all WT were sensitive to doxorubicin with $\mathrm{IC}_{50} \mathrm{~S}$ in the nanomolar range $(\sim 20-500 \mathrm{nM})$ as

221 compared to the normal cell lines which had an IC50 range of 0.65-1.5uM (Fig 4a).

223 We then evaluated the effect of KPT-330 and doxorubicin treatment in combination on cell viability as

224 measured by CellTiter-Glo. The addition of KPT-330 to doxorubicin significantly enhanced cell death in

225 WT cells (Fig $\mathbf{4 b}$ ). We then performed synergy experiments (Methods) to determine if this combination

226 was synergistic or additive. Across multiple concentrations of KPT-330 and doxorubicin in all of the WT

227 cell lines tested, the combination was synergistic (e.g., scores $>10$; Fig $\mathbf{4 c - d}$ ). In contrast, this combination

228 was antagonistic in normal cells (Fig 4d). Collectively, we found a synergistic interaction between KPT-

229330 and doxorubicin in FHWT as compared to normal kidney cell lines.

231 We followed up these in vitro studies with in vivo studies to further validate the potential synergistic effect 232 of KPT-330 and doxorubicin. Of our patients samples, we were able to generate two PDXs from 233 CCLF_PEDS_0041 and CCLF_PEDS_0023. Given that CCLF_PEDS_0023 has features of anaplastic 234 Wilms, we performed our in vivo studies with the PDX from CCLF_PEDS_0041, a patient with FHWT. 235 Interestingly, the CCLF_PEDS_0041_T1 cell line and cell line derived from the PDX 236 (CCLF_PEDS_0041_T2) were some of the least sensitive WT cell lines to KPT-330 and doxorubicin 237 treatments (Fig 2d and 4a-b). We treated tumor xenografts with placebo, doxorubicin, KPT-330 or the 
Mittal and Lee et. al.

combination of doxorubicin and KPT-330 for 28 days and then monitored tumor growth until endpoints were reached or after 150 days following treatment initiation. We found at 22 days, that KPT-330 led to $49 \%$ decrease $(p$-value $=0.046)$, doxorubicin led to $87 \%$ decrease $(p$-value $<0.005)$ and the combination

241 led to $99 \%$ decrease ( -value $<0.005$ ) in tumor volume compared to the vehicle $(\mathbf{F i g} \mathbf{4 e})$. We then assessed

242 the effects following cessation of therapeutic treatment. We found that monotherapy with KPT-330 or

243 doxorubicin led to a median survival of 39 and 53.5 days, respectively (Fig $\mathbf{4 f - g}$ ). Further, the combination

244 of KPT-330 and doxorubicin had an undefined median survival with five of eight mice exhibiting a

245 complete response (Fig 4f-g). Taken together, these observations suggest that inhibition of XPO1 in

246 combination with doxorubicin chemotherapy are synergistic in vivo.

\section{DISCUSSION}

250 Here we have developed short term cell line models of Wilms Tumor (Fig 1). Although these cell lines, 251 which maintain a faithful representation of their genomics and transcriptomics, only grow for a finite 252 number of passages, we show that they can be used to perform systematic loss-of-function studies (Fig 2).

253 We find that integration of targeted RNA interference and CRISPR-Cas9 screens identifies the nuclear 254 export apparatus as a therapeutic target in FHWT.

256 XPO1 regulates a spectrum of cellular processes by controlling the active transport of over 200 proteins out 257 of the nucleus, including tumor suppressors (TSs) and transcription factors (TFs) (such as TOP2A, p53, 258 WTX, APC, Rb, and PI3K/AKT) ${ }^{3}$. Due to its important physiological role, inhibition of XPO1 by the small 259 molecule inhibitor, KPT-330 (selinexor), is widely being tested in the phase I and phase II clinical trials in 260 multiple malignancies and has FDA approval in several hematologic malignancies ${ }^{4-6}$. As part of the 261 Pediatric Preclinical Testing Program, KPT-330 was tested as a single agent across a panel of pediatric 262 hematologic and solid tumor PDXs which included several WT xenografts ${ }^{51}$. In this study, the group used 263 KPT-330 in 3 WT PDXs. They found one PDX (KT-10) had maintained a complete response at 42 days 
Mittal and Lee et. al.

264 post-treatment initiation while the other two had partial responses. However, mechanisms and biomarkers 265 remain unknown.

267 Here, we show that inhibition of nuclear export leads to primarily a G2/M arrest in FHWT and loss of TP53

268 leads to significant resistance to KPT-330 (Fig 3). While the role of TP53 in response to KPT-330 has been

269 previously reported ${ }^{52}$, we identified that suppression or inhibition of nuclear export leads to suppression of

270 TRIP13 in FHWT. We find that although TRIP13 is traditionally thought to affect $\mathrm{G} 2 / \mathrm{M}^{53,54}$, loss of TRIP13

271 affects both G1 and G2/M in FHWT. Furthermore, when we compare the differentially expressed genes

272 between KPT-330 treated cells as compared to cells with TRIP13 suppression, we find several potential

273 tumor suppressors (e.g. $C D H 1, M Y C T 1$ ) and $S A M D 9$, a gene having antiproliferative properties, whose

274 expression increases. Future studies will explore these findings to better elucidate the function of TRIP13

275 in FHWT. Finally, we show that there is significant synergy when KPT-330 is combined with the 276 topoisomerase II inhibitor, doxorubicin, in vivo where five of eight mice achieved cure following a single 277 cycle of combination therapy (Fig 4).

279 Future work requires the development of additional 1q gain or 1p/16q loss of heterozygosity (LOH) in vitro 280 models. In addition, our data from one patient derived model with DAWT (TP53 mutant) suggests that 281 inhibition of nuclear export may be a therapeutic strategy but additional models and validation studies are 282 needed (Fig 2b).

284 Taken together, we have identified that KPT-330 acts in part by inhibiting TRIP13 in Wilms Tumor. In 285 addition, suppression of TRIP13 may serve as a biomarker of response in WT. Finally, in combination with 286 a topoisomerase II inhibitor, nuclear export inhibitors could be studied in patients with high-risk Favorable 287 Histology WT. 
Mittal and Lee et. al.

\section{METHODS}

\section{Development of patient-derived Wilms tumor cell lines}

292 Tumor cells were isolated from tumor and if available, adjacent normal kidneys tissues of patients with a 293 pathological diagnosis of Wilms Tumor. Samples were obtained under IRB approved protocols from 294 patients at either Dana Farber / Boston Children's Cancer and Blood Disorders Center or Aflac Cancer and 295 Blood Disorders Center at the Children's Healthcare of Atlanta and Emory University School of Medicine.

296 Tumor tissue or adjacent normal tissue was first minced into $1-2 \mathrm{~mm}^{3}$ pieces and resuspended in F-media 297 as previously described ${ }^{23}$. Samples were then plated in six-well plates. Cells were serially passaged after 298 reaching confluency of 70-80\%. For PDX cell lines, a tumor fragment $(<5 \mathrm{~mm}$ in diameter) was implanted 299 into NSG mice and once passaged twice, a tumor fragment was then minced and fragmented on a 6 well 300 dish to develop a PDX cell line. In general Wilms Tumor cells were grown for up to 30 passages and normal 301 kidney cells were grown for approximately 12-15 passages before senescing.

\section{Low coverage whole genome sequencing (WGS) and Copy Number Analyses}

304 DNA from tumor tissue, normal tissue if available or blood, and cell pellets from our patient-derived cell 305 lines was extracted (NEB, T3010S). Genomic libraries were prepared (Illumina) by Novogene Inc and 306 sequenced at $0.1 \mathrm{x}$ on Novoseq 6000 (Illumina). Fastq or BAM files were mapped and aligned using 307 Illumina Dragen v3.7.5 to GrCh38 on Amazon Web Services. ichorCNA v0.10 was then used to determine 308 copy number alterations ${ }^{55}$. Copy number variations (amplification, gain, deletion) were calculated by 309 ichorCNA.

\section{Whole exome sequencing}

312 WES was performed using DNA as described above. For five patients, WES was performed at the Broad 313 Institute Genomics Platform using HiSeq 2000 (Illumina). WES libraries were based on an Illumina

314 Customized Exon (ICE) array. For three patients, WES was performed at Novogene Inc using Novoseq6000

315 (Illumina). Libraries were based on an Agilent array. Sample coverage was $>100 \mathrm{x}$ for tumor and $>50 \mathrm{x}$ for 
Mittal and Lee et. al.

normal. Fastq or BAM files were mapped and aligned using Illumina Dragen v3.7.5 to GrCh38 ${ }^{56}$ on Amazon Web Services. Variant call files (vcf) were subsequently processed with OpenCRAVAT using cancer hostpots, cancer gene landscape, chasm plus, civic, cosmic, mutpanning and ndex NCI to filter for

319 pathogenic variants.

\section{RNA sequencing}

322 RNA was extracted from samples collected from tumor, adjacent normal and cell lines (Qiagen RNeasy or 323 NEB Monarch Total RNA). Libraries were prepared using Illumina TruSeq. Samples were run with at least 32450 million paired-end reads using Novoseq 6000 (Illumina). For mechanistic studies, RNA was collected 325 in biological replicates from cells treated with shRNAs or compounds as listed in the figures. Similar 326 methods were used in sequencing with the exception that samples were sequenced at 20 million single-end 327 reads per sample. Fastq or BAM files were mapped and aligned using Illumina Dragen v3.7.5 to GrCh38 328 and GenCode $36^{57}$ on Amazon Web Services. Samples were then quantified using salmon through Illumina 329 Dragen v3.7.5. The quantification files were imported into R using tximport ${ }^{58}$ and ComBat-seq ${ }^{59}$ was used 330 to correct for batch effects. Differential gene expression analysis was then performed using DESeq2 ${ }^{60}$. $331 \mathrm{UMAP}^{61}$ was used for visualizing clustering between samples.

\section{Loss of function screens - methodology and analyses}

334 RNAi and CRISPR-Cas9 screens were performed as previously described ${ }^{21-23}$. Specifically, we transduced 335 into noted cell lines the DCT v1.0 libraries: shRNA (CP1050) and sgRNA (CP0026) libraries from the 336 Broad Institute Genetic Perturbation Platform (GPP) (http://www.broadinstitute.org/rnai/public/). In 337 parallel, CCLF_PEDS_0023_T, CCLF_PEDS_0023_T2, and CCLF_PEDS_0002_T cells were transduced 338 with Cas9 expression vector pLX311_Cas ${ }^{62}$. These stable cells were subsequently transduced using the 339 DCT v2.0 libraries and at a multiplicity of infection (MOI) between $0.3-0.6^{32}$. Screens were performed goal 340 representation rate $>500$ cells per shRNA or sgRNA. Cells were passaged every 5-7 days until days 25-29 
Mittal and Lee et. al.

341 following transduction. Genomic DNA was extracted from an early time point and at the end time point.

342 Samples were sequenced as previously described, deconvoluted and analyzed using MAGeK ${ }^{33}$.

\section{Cell viability assays}

345 Cells were plated in a concentration of 2,500 cells/well in a 96 well plate (Corning, 3903, or Greiner Bio-

346 One, 655098) and were incubated overnight in F-media overnight. After 24 hours, F-media was aspirated

347 and F-media with the indicated drug concentration was added. Cells were treated between range of 0.007

$348 \mu \mathrm{M}$ to $50 \mu \mathrm{M}$ of KPT-330 (Selleck, S7252) and doxorubicin (Selleck, S1208) for 72 hours. Cell viability

349 was measured using CellTiter-Glo (Promega, G7573). $\mathrm{IC}_{50}$ S of KPT-330 and doxorubicin were determined

350 in each cell line by fitting the dose response curve using Graphpad Prism v9.3.0. Each experiment was

351 repeated in at least two biological replicates.

353 XPO1 and TRIP13 shRNA experiments

354 shRNA sequences were designed using the Broad Institute GPP portal

355 (http://www.broadinstitute.org/rnai/public/). Oligos were obtained from Integrated DNA Technologies

356 (IDT). Oligos were annealed and ligated with pLKO.5 as previously described. Constructs were transfected

357 using TransIT-LT1 (Mirus Bio LLC, Madison, WI, USA) and HEK 293T cells. Cells were then transduced

358 with lentivirus to achieve appropriate knockdown without significant viral toxicity as previously described

35922 . Following selection with puromycin (Invivogen), cell proliferation was assayed by CellTiter-Glo at 10

360 days. Experiments were repeated a minimum of three times in technical triplicates. shRNA sequences can

361 be found in Supp Table 4.

\section{TP53 CRISPR- Cas9 deletion}

364 CCLF_PEDS1012T and CCLF_PEDS_0022_T cells were transduced with Cas9 expression vector 365 pLX311_Cas9. These cells were subsequently transduced with sgTP53-1 and sgLacZ in the pXPR003 
Mittal and Lee et. al.

backbone. Transduced cells were then cultured in the indicated antibiotics for selection and suppression of

367 gene expression was confirmed by immunoblotting.

\section{qRT-PCR}

370 Total RNA was extracted using the NEB Monarch RNA extraction kit. One microgram of RNA and oligo

371 primers were used for cDNA synthesis in a total reaction volume of $20 \mu \mathrm{L}$ High-Capacity cDNA Reverse

372 Transcription Kit (Thermo Fisher Scientific, 4368814). qRT-PCR reactions were prepared using SYBR-

373 Green PCR Master Mix (ThermoFisher Scientific, 4367659) and run on a BioRad CFX96 qPCR System

374 with a minimum of technical duplicates. Relative mRNA levels were calculated using the 2- $\Delta \Delta \mathrm{Ct}_{\text {method }}{ }^{63}$.

375 Sequences of primers used in qRT-PCR experiments are detailed in Supp Table 3. Results shown are 376 representative of at least two biological replicates.

\section{Immunoblots}

379 Cells were grown to $70-80 \%$ confluency and were treated or transduced as indicated the figures. Thereafter, 380 cells were lysed using 1x RIPA (Cell Signaling Technologies, 9806) with protease inhibitors (coMplete, 381 Roche, 42484600) and phosphatase inhibitors (PhosSTOP, Roche, 04906837001). For experiments using 382 nuclear and cytoplasmic fractions, fractions were extracted using NE-PER (ThermoFisher Scientific, 383 78835). Using $10 \%$ or 4-12\% SDS-PAGE gels, gels were transferred onto PVDF (Millipore, IPFL00010) 384 or nitrocellulose membranes (ThermoFisher Scientific, IB23001). Blots were then visualized using 385 Odyssey CLx (Licor, Lincoln, NE). Antibodies used in this study include: XPO1 (Santa Cruz; sc-5595), $\beta$ 386 Actin (C-4) (Santa Cruz; sc-47778), $\beta$-Actin (Cell Signaling; 8457), TP53 (Santa Cruz; sc-123), TRIP13 387 (Abcam; ab128171), $\alpha$-Tubulin (Santa Cruz, sc-5286), $\alpha$-Tubulin (Cell Signaling; 2144), Lamin A/C (Cell 388 Signaling; 4777 or 2032), p21 (Cell Signaling; 29475), CCND1 (Santa Cruz; sc-8396). Results shown are 389 representative of at least two biological replicates. 


\section{Mittal and Lee et. al.}

392 WT cells were plated in 96 well plates at a concentration of 2,500 cells/well. After 24 hours, cells were 393 treated with a combination of KPT330 and doxorubicin in a $5 \times 5$ matrix on the same microtiter plate in 394 duplicate. Each plate included a 4-dose dilution of each drug alone or in combination and included DMSO 395 controls. Following a 3-day incubation, we performed a cell viability assay using CellTiter-Glo. Relative

396 cell viabilities were then calculated and analyzed with SynergyFinder ${ }^{64}$ using two alternate drug-drug 397 interaction models: zero interaction potency (ZIP) ${ }^{65}$ and bliss independence ${ }^{66}$. Experiments were performed 398 in biological replicates or triplicates.

$400 \quad$ In vivo experiments

401 Female NSG (NOD-scid IL2Rgamma null) mice, 6-weeks old were purchased from The Jackson 402 Laboratory (Bar Harbor, ME). Animals were acclimated for at least 5 days before initiation of the study. 403 The study was conducted at Dana-Farber Cancer Institute (DFCI) with the approval of the Institutional 404 Animal Care and Use Committee in an AAALAC accredited vivarium.

NSG mice were implanted with CCLF_PEDS_0041_T2 tumor fragments from previously expanded

407 tumors, subcutaneously in the hind-flank. Tumors were allowed to establish to $86-163 \mathrm{~mm} 3$ (average $408114.3 \mathrm{~mm} 3$ ) in size before randomization using Studylog software (San Francisco, CA) into various 409 treatment groups with 8 mice/group as follows: vehicle control (0.6\% Pluronic F-68 + 0.6\% Plasdone PVP; 410 oral gavage on MWF x 4 weeks), selinexor (purchased from Selleck Chemicals LLC, 15 mg/kg oral gavage 411 on MWF x 4 weeks), doxorubicin (obtained from DFCI pharmacy, $5 \mathrm{mg} / \mathrm{kg}$ intravenous injection on days

4122 and 9) and the combination of selinexor and doxorubicin. No statistical methods were used to pre413 determine sample sizes but our sample sizes are similar to those reported in previous publications ${ }^{22,23,67}$.

414 Once the treatment was completed, tumors were monitored at least once a week. For single agent and 415 combination agent efficacy studies, mice were dosed for 21 or 28 days and monitored daily. Drugs were 416 then withdrawn, and tumors were monitored twice weekly until study termination on day 150 . Tumor 417 volumes were calculated using the following formula: $(\mathrm{mm} 3)=$ length $\times$ width $\times$ width $\times 0.5$. Mice were 
Mittal and Lee et. al.

418 immediately euthanized if the tumor volume exceeded $2000 \mathrm{~mm} 3$ or if the tumors became necrotic or

419 ulcerated. The compounds were well tolerated with less than $8 \%$ body weight loss. Data collection and 420 analysis were not performed blind to the conditions of the experiments. GraphPad Prism v9.3.0 was used 421 to calculate significance in the Kaplan-Meier curves.

\section{AVAILABILITY OF DATA AND RESOURCES}

425 Sequencing data reported in this paper has been deposited in the database _ PENDING_.

\section{COMPETING INTERESTS}

429 The authors declare the following competing financial interests - K.M. is currently employed at PreludeDx.

430 A.L.C. is currently an employee of Aztra Zeneca. G.C. is currently an employee of Johnson \& Johnson.

431 D.M.W is currently an employee of Merck and has research support from Daiichi Sankyo, Abcuro,

432 Verastem, Secura, and is on the Advisory Board or has equity in Ajax, Travera, Astra Zeneca, Bantam. K.S.

433 receives grant funding from Novartis and KronosBio, consults for and has stock options in Auron

434 Therapeutics and has served as an advisor for KronosBio and AstraZeneca. W.C.H. is a consultant for 435 Thermo Fisher, Solasta Ventures, MPM Capital, KSQ Therapeutics, iTeos, Tyra Biosciences, Jubilant 436 Therapeutics, RAPPTA Therapeutics, Function Oncology, Frontier Medicines and Calyx. The remaining 437 authors declare that they have no competing interests.

\section{FUNDING}

441 Research reported in this publication was supported by the following: NIGMS T32GM008490 (GWC), 442 T32GM007739 (WJK), NCI K08 CA2555569-01 (AJM), NCI R35 CA231958 (DMW), NIH R35 
Mittal and Lee et. al. Cancer YIA (ALH), Rally for Childhood Cancer Investigator Grant 21IN12 (ALH).

\section{AUTHOR CONTRIBUTIONS}

$448 \mathrm{KM}, \mathrm{WCH}$ and $\mathrm{ALH}$ in the design of the study. KM, BPL, WCH and ALH wrote the manuscript. KM, 449 BPL, GWC, BD, MR, MB and ALH performed analyses of the sequencing data. KM, BPL, WJK, MD, BK, 450 PK, MT, JB, ALH generated the cancer cell lines. JS, HCJ, TL and KCG oversaw the Aflac Solid Tumor 451 Biorepository. SM, CC, and BC oversaw the DF/BCH Solid Tumor Biorepository. WJK, MD, DA, MK, $452 \mathrm{XY}, \mathrm{GC}, \mathrm{DR}$ and ALH performed and/or analyzed the functional genomic screens. ALC, BL, CM, AJM, 453 KL, DMW, PG generated the PDXs and performed in vivo studies. MB, BC, MT, JB, KL, DR, AJM, DMW, 454 PG, JS, MR, EAM, KS, KCG, WCH and ALH supervised the studies. All authors discussed the results and 455 implications and edited the manuscript. All authors also read and approved the final manuscript.

\section{ACKNOWLEDGEMENTS}

459 We thank the patients and their families for their participation. We thank the Hong, Spangle and Hahn labs 460 for their thoughtful comments and suggestions. Tissue samples were provided by the Children's Healthcare 461 of Atlanta Pediatric Bio-Repository or Dana-Farber/Boston Children's Center for Cancer and Blood 462 Disorders. Other investigators may have received specimens from the same subjects. Research reported in 463 this publication was supported in part by the Pediatrics/Winship Flow Cytometry Core of Winship Cancer 464 Institute of Emory University, Children's Healthcare of Atlanta and NIH/NCI under award number 465 P30CA138292, by the Bioinformatics and Systems Biology Shared and the Biostatistics Shared Resource 466 of Winship Cancer Institute of Emory University and NIH/NCI under award number P30CA138292. The 467 content is solely the responsibility of the authors and does not necessarily represent the official views of 468 the National Institutes of Health. 
Mittal and Lee et. al.

\section{References}

1. Nakata, K. et al. Incidence of childhood renal tumours: An international population-based study. Int J Cancer 147, 3313-3327 (2020).

2. Smith, M.A., Altekruse, S.F., Adamson, P.C., Reaman, G.H. \& Seibel, N.L. Declining childhood and adolescent cancer mortality. Cancer 120, 2497-506 (2014).

3. Spreafico, F. et al. Wilms tumour. Nat Rev Dis Primers 7, 75 (2021).

4. Wilms Tumor - Childhood: Statistics. Vol. 2021 (cancer.net, 2021).

5. Axt, J. et al. Race disparities in Wilms tumor incidence and biology. $J$ Surg Res 170, 112 9 (2011).

6. $\quad$ Siegel, R.L., Miller, K.D., Fuchs, H.E. \& Jemal, A. Cancer Statistics, 2021. CA Cancer J Clin 71, 7-33 (2021).

7. Daw, N.C. et al. Activity of Vincristine and Irinotecan in Diffuse Anaplastic Wilms Tumor and Therapy Outcomes of Stage II to IV Disease: Results of the Children's Oncology Group AREN0321 Study. J Clin Oncol 38, 1558-1568 (2020).

8. Fernandez, C.V. et al. Outcome and Prognostic Factors in Stage III Favorable-Histology Wilms Tumor: A Report From the Children's Oncology Group Study AREN0532. J Clin Oncol 36, 254-261 (2018).

9. Jain, J., Sutton, K.S. \& Hong, A.L. Progress Update in Pediatric Renal Tumors. Curr Oncol Rep 23, 33 (2021).

10. Garvin, A.J., Re, G.G., Tarnowski, B.I., Hazen-Martin, D.J. \& Sens, D.A. The G401 cell line, utilized for studies of chromosomal changes in Wilms' tumor, is derived from a rhabdoid tumor of the kidney. Am J Pathol 142, 375-80 (1993).

11. Pritchard-Jones, K. \& Perotti, D. WARNING: G-401 and SK-NEP-1 cell lines are not Wilms tumor cell lines. Pediatr Blood Cancer 66, e27741 (2019).

12. Smith, M.A. et al. SK-NEP-1 and Rh1 are Ewing family tumor lines. Pediatr Blood Cancer 50, 703-6 (2008).

13. Stroup, E. et al. WT-CLS1 is a rhabdoid tumor cell line and can be inhibited by miR-16. Cancer Reports 2, e1110 (2018).

14. Brandt, A. et al. Establishment of a Conditionally Immortalized Wilms Tumor Cell Line with a Homozygous WT1 Deletion within a Heterozygous 11p13 Deletion and UPD Limited to 11p15. PLoS One 11, e0155561 (2016).

15. Royer-Pokora, B. et al. Wilms tumor cells with WT1 mutations have characteristic features of mesenchymal stem cells and express molecular markers of paraxial mesoderm. Hum Mol Genet 19, 1651-68 (2010).

16. Royer-Pokora, B. et al. Comprehensive Biology and Genetics Compendium of Wilms Tumor Cell Lines with Different WT1 Mutations. Cancers (Basel) 13(2020).

17. Wegert, J. et al. Characterization of primary Wilms tumor cultures as an in vitro model. Genes Chromosomes Cancer 51, 92-104 (2012).

18. Calandrini, C. et al. An organoid biobank for childhood kidney cancers that captures disease and tissue heterogeneity. Nature Communications 11, 1310 (2020).

19. Murphy, A.J. et al. Forty-five patient-derived xenografts capture the clinical and biological heterogeneity of Wilms tumor. Nature Communications 10, 5806 (2019).

20. Ooms, A.H.A.G., Calandrini, C., de Krijger, R.R. \& Drost, J. Organoid models of childhood kidney tumours. Nature Reviews Urology 17, 311-313 (2020).

21. Dharia, N.V. et al. A first-generation pediatric cancer dependency map. Nature Genetics 53, 529-538 (2021). 
Mittal and Lee et. al.

22. Hong, A.L. et al. Integrated genetic and pharmacologic interrogation of rare cancers. Nature Communications 7, 11987 (2016).

23. Hong, A.L. et al. Renal medullary carcinomas depend upon SMARCB1 loss and are sensitive to proteasome inhibition. Elife 8(2019).

24. Chagtai, T. et al. Gain of 1q As a Prognostic Biomarker in Wilms Tumors (WTs) Treated With Preoperative Chemotherapy in the International Society of Paediatric Oncology (SIOP) WT 2001 Trial: A SIOP Renal Tumours Biology Consortium Study. J Clin Oncol 34, 3195-203 (2016).

25. Gratias, E.J. et al. Association of Chromosome 1q Gain With Inferior Survival in Favorable-Histology Wilms Tumor: A Report From the Children's Oncology Group. J Clin Oncol 34, 3189-94 (2016).

26. Ooms, A.H. et al. Significance of TP53 Mutation in Wilms Tumors with Diffuse Anaplasia: A Report from the Children's Oncology Group. Clin Cancer Res 22, 5582-5591 (2016).

27. Melville, L.M.a.J.H.a.J. UMAP: Uniform Manifold Approximation and Projection for Dimension Reduction. (2020).

28. Gadd, S. et al. A Children's Oncology Group and TARGET initiative exploring the genetic landscape of Wilms tumor. Nat Genet 49, 1487-1494 (2017).

29. Murphy, A.J. et al. SIX2 and CITED1, markers of nephronic progenitor self-renewal, remain active in primitive elements of Wilms' tumor. J Pediatr Surg 47, 1239-49 (2012).

30. Walz, A.L. et al. Recurrent DGCR8, DROSHA, and SIX homeodomain mutations in favorable histology Wilms tumors. Cancer Cell 27, 286-97 (2015).

31. Wegert, J. et al. Mutations in the SIX1/2 pathway and the DROSHA/DGCR8 miRNA microprocessor complex underlie high-risk blastemal type Wilms tumors. Cancer Cell 27, 298-311 (2015).

32. Harris, I.S. et al. Deubiquitinases Maintain Protein Homeostasis and Survival of Cancer Cells upon Glutathione Depletion. Cell Metab 29, 1166-1181.e6 (2019).

33. Li, W. et al. MAGeCK enables robust identification of essential genes from genome-scale CRISPR/Cas9 knockout screens. Genome Biol 15, 554 (2014).

34. Kasamon, Y.L. et al. FDA Approval Summary: Selinexor for Relapsed or Refractory Diffuse Large B-Cell Lymphoma. Oncologist 26, 879-886 (2021).

35. Bjork, I., Peralez, J., Haussler, D., Spunt, S.L. \& Vaske, O.M. Data sharing for clinical utility. Cold Spring Harb Mol Case Stud 5(2019).

36. Turner, J.G. et al. CRM1 Inhibition Sensitizes Drug Resistant Human Myeloma Cells to Topoisomerase II and Proteasome Inhibitors both In Vitro and Ex Vivo. J Cancer 4, 61425 (2013).

37. Azmi, A.S., Uddin, M.H. \& Mohammad, R.M. The nuclear export protein XPO1 - from biology to targeted therapy. Nat Rev Clin Oncol 18, 152-169 (2021).

38. Gravina, G.L. et al. KPT-330, a potent and selective exportin-1 (XPO-1) inhibitor, shows antitumor effects modulating the expression of cyclin D1 and survivin [corrected] in prostate cancer models. BMC Cancer 15, 941 (2015).

39. Subramanian, A. et al. Gene set enrichment analysis: a knowledge-based approach for interpreting genome-wide expression profiles. Proc Natl Acad Sci U S A 102, 15545-50 (2005). 
Mittal and Lee et. al.

40. Etchin, J. et al. KPT-330 inhibitor of CRM1 (XPO1)-mediated nuclear export has selective anti-leukaemic activity in preclinical models of T-cell acute lymphoblastic leukaemia and acute myeloid leukaemia. Br J Haematol 161, 117-27 (2013).

41. Pressly, J.D., Hama, T., Brien, S.O., Regner, K.R. \& Park, F. TRIP13-deficient tubular epithelial cells are susceptible to apoptosis following acute kidney injury. Sci Rep 7, 43196 (2017).

42. Yost, S. et al. Biallelic TRIP13 mutations predispose to Wilms tumor and chromosome missegregation. Nat Genet 49, 1148-1151 (2017).

43. Buehler, E., Chen, Y.C. \& Martin, S. C911: A bench-level control for sequence specific siRNA off-target effects. PLoS One 7, e51942 (2012).

44. Lara, M.F. et al. Gene profiling approaches help to define the specific functions of retinoblastoma family in epidermis. Mol Carcinog 47, 209-21 (2008).

45. Fu, S. et al. MYCT1-TV, a novel MYCT1 transcript, is regulated by c-Myc and may participate in laryngeal carcinogenesis. PLoS One 6, e25648 (2011).

46. Nagamachi, A. et al. Haploinsufficiency of SAMD9L, an endosome fusion facilitator, causes myeloid malignancies in mice mimicking human diseases with monosomy 7. Cancer Cell 24, 305-17 (2013).

47. Thanh Nha Uyen, L. et al. PCDH17 functions as a common tumor suppressor gene in acute leukemia and its transcriptional downregulation is mediated primarily by aberrant histone acetylation, not DNA methylation. Int J Hematol 111, 451-462 (2020).

48. Bond, J. et al. ASPM is a major determinant of cerebral cortical size. Nat Genet 32, 31620 (2002).

49. Li, H. et al. CDCA7 Facilitates Tumor Progression by Directly Regulating CCNA2 Expression in Esophageal Squamous Cell Carcinoma. Front Oncol 11, 734655 (2021).

50. Breslow, N.E. et al. Doxorubicin for favorable histology, Stage II-III Wilms tumor: results from the National Wilms Tumor Studies. Cancer 101, 1072-80 (2004).

51. Attiyeh, E.F. et al. Pharmacodynamic and genomic markers associated with response to the XPO1/CRM1 inhibitor selinexor (KPT-330): A report from the pediatric preclinical testing program. Pediatr Blood Cancer 63, 276-86 (2016).

52. Sun, H. et al. CRM1 Inhibition Promotes Cytotoxicity in Ewing Sarcoma Cells by Repressing EWS-FLI1-Dependent IGF-1 Signaling. Cancer Res 76, 2687-97 (2016).

53. Hama, T. et al. DNA damage is overcome by TRIP13 overexpression during cisplatin nephrotoxicity. JCI Insight 6(2021).

54. Sheng, N. et al. TRIP13 promotes tumor growth and is associated with poor prognosis in colorectal cancer. Cell Death Dis 9, 402 (2018).

55. Adalsteinsson, V.A. et al. Scalable whole-exome sequencing of cell-free DNA reveals high concordance with metastatic tumors. Nat Commun 8, 1324 (2017).

56. Schneider, V.A. et al. Evaluation of GRCh38 and de novo haploid genome assemblies demonstrates the enduring quality of the reference assembly. bioRxiv, 072116 (2016).

57. Harrow, J. et al. GENCODE: the reference human genome annotation for The ENCODE Project. Genome Res 22, 1760-74 (2012).

58. Soneson, C., Love, M.I. \& Robinson, M.D. Differential analyses for RNA-seq: transcriptlevel estimates improve gene-level inferences. F1000Res 4, 1521 (2015).

59. Zhang, Y., Parmigiani, G. \& Johnson, W.E. ComBat-seq: batch effect adjustment for RNAseq count data. NAR Genom Bioinform 2, lqaa078 (2020). 
Mittal and Lee et. al.

60. Love, M.I., Huber, W. \& Anders, S. Moderated estimation of fold change and dispersion for RNA-seq data with DESeq2. Genome Biol 15, 550 (2014).

61. McInnes, L., Healy, J. \& Melville, J. Umap: Uniform manifold approximation and projection for dimension reduction. arXiv preprint arXiv:1802.03426 (2018).

62. Giacomelli, A.O. et al. Mutational processes shape the landscape of TP53 mutations in human cancer. Nature Genetics 50, 1381-1387 (2018).

63. Livak, K.J. \& Schmittgen, T.D. Analysis of relative gene expression data using real-time quantitative PCR and the 2(-Delta Delta C(T)) Method. Methods 25, 402-8 (2001).

64. Ianevski, A., He, L., Aittokallio, T. \& Tang, J. SynergyFinder: a web application for analyzing drug combination dose-response matrix data. Bioinformatics 33, 2413-2415 (2017).

65. Yadav, B., Wennerberg, K., Aittokallio, T. \& Tang, J. Searching for Drug Synergy in Complex Dose-Response Landscapes Using an Interaction Potency Model. Comput Struct Biotechnol J 13, 504-13 (2015).

66. BLISS, C.I. THE TOXICITY OF POISONS APPLIED JOINTLY1. Annals of Applied Biology 26, 585-615 (1939).

67. Bandopadhayay, P. et al. Neuronal differentiation and cell-cycle programs mediate response to BET-bromodomain inhibition in MYC-driven medulloblastoma. Nat Commun 10, 2400 (2019). 
bioRxiv preprint doi: https://doi.org/10.1101/2022.02.23.481521; this version posted February 25, 2022. The copyright holder for this preprint (which was not certified by peer review) is the author/funder. All rights reserved. No reuse allowed without permission.

Mittal and Lee et. al.

\section{FIGURES AND FIGURE LEGENDS}


bioRxiv preprint doi: https://doi.org/10.1101/202: 0 (which was not certified by peer revtew) is the auth pr/funder. All rights reserved. No re e allowed without permission.

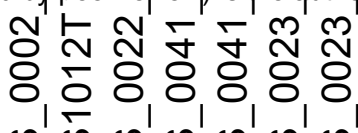

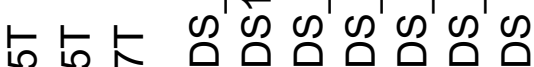

๘尺

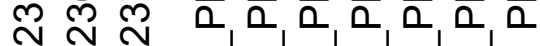

u v

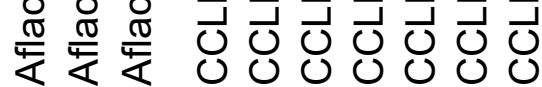

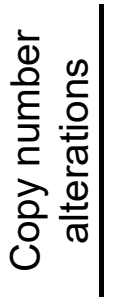

Relapse

Anaplasia

Model type
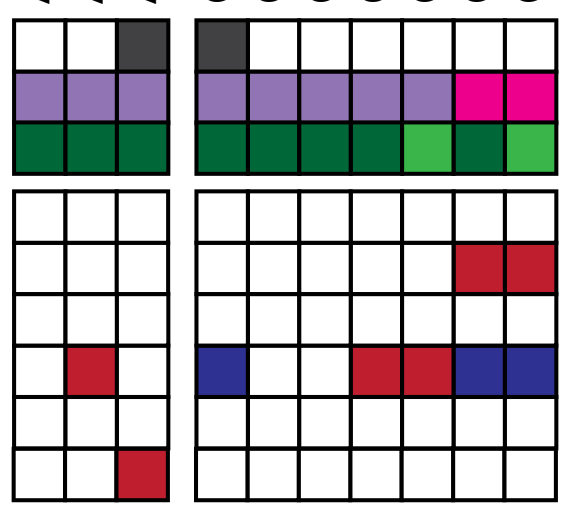

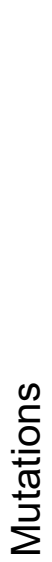

b

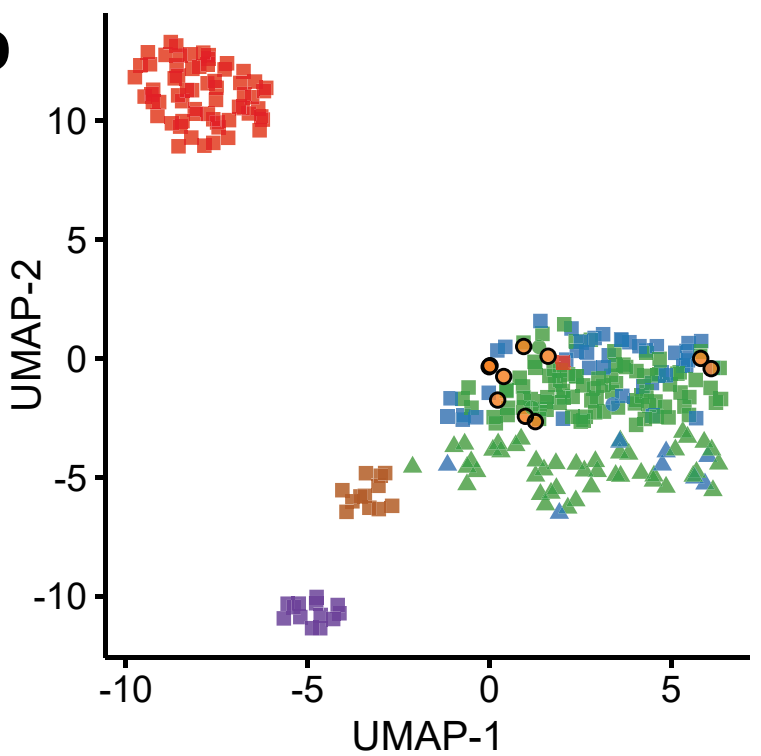

Group
- Wilms tumor cell line

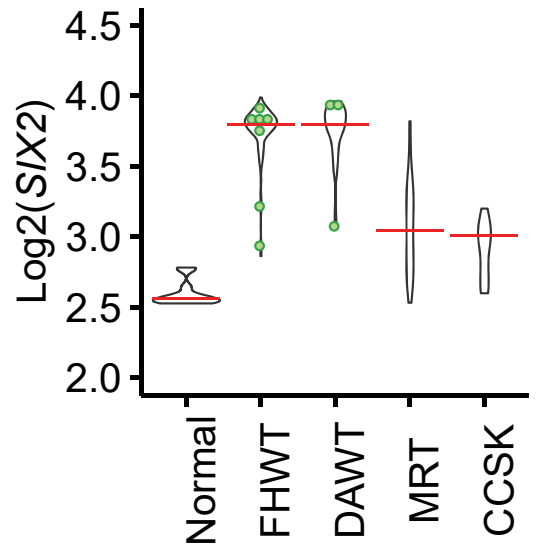

d

- Wilms tumor cell line

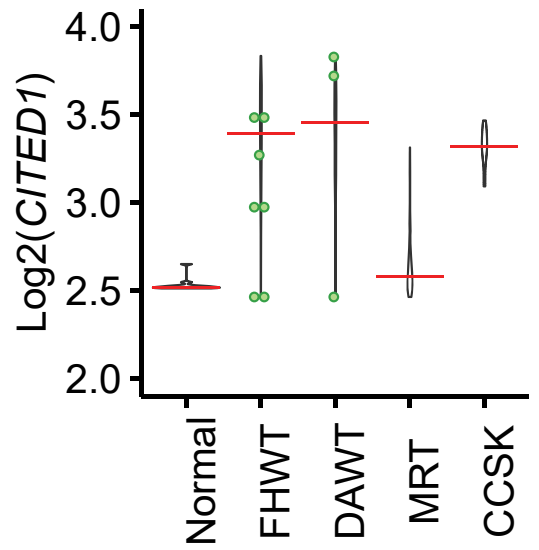

- This study

- TARGET

A St. Jude

Diagnosis

- Normal

- FHWT

- DAWT

O Wilms tumor cell line

- Clear cell sarcoma of kidney

- Malignant rhabdoid tumor 
Mittal and Lee et. al.

626 Figure 1: Overview of the Genomic and transcriptomic analysis of Wilms tumor cells lines: (a) Co-

627 mutation plot representing the clinicopathological information (top panel), copy number alterations (middle

628 panel), and mutations (bottom panel) in the WT cell lines. T or T1 represents the cell line derived from the

629 patient while T2 represents the cell line derived from the first passage PDX. (b) Two-dimensional

630 representation of RNA-seq data using uniform manifold approximation and projection (UMAP)

631 demonstrates high concordance between TARGET (squares) and St. Jude primary (triangles) WT (green -

632 FHWT and blue - DAWT) and our Wilms tumor cell lines (orange circles with black boundaries). Normal

633 tissues samples, clear cell sarcoma of the kidney, and malignant rhabdoid tumor all were clustered

634 separately. (c) and (d) Dot plots representing the higher expression of commonly dysregulated genes (SIX2

635 and CITED1) in WT in the TARGET and St. Jude datasets and WT cell lines. Green dots indicate WT cell

636 lines. 

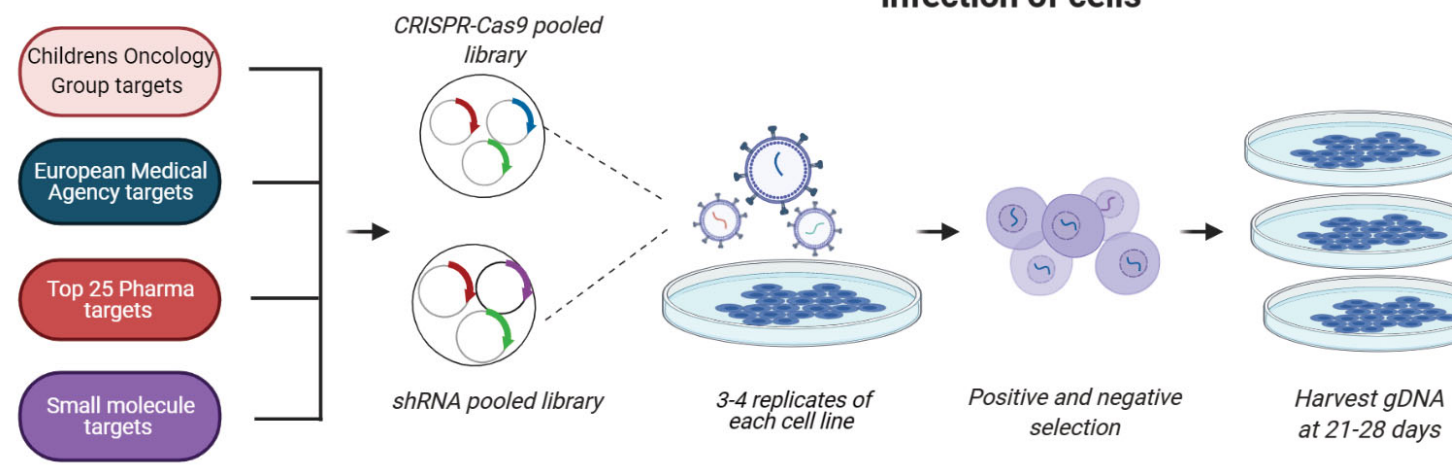

RNAi
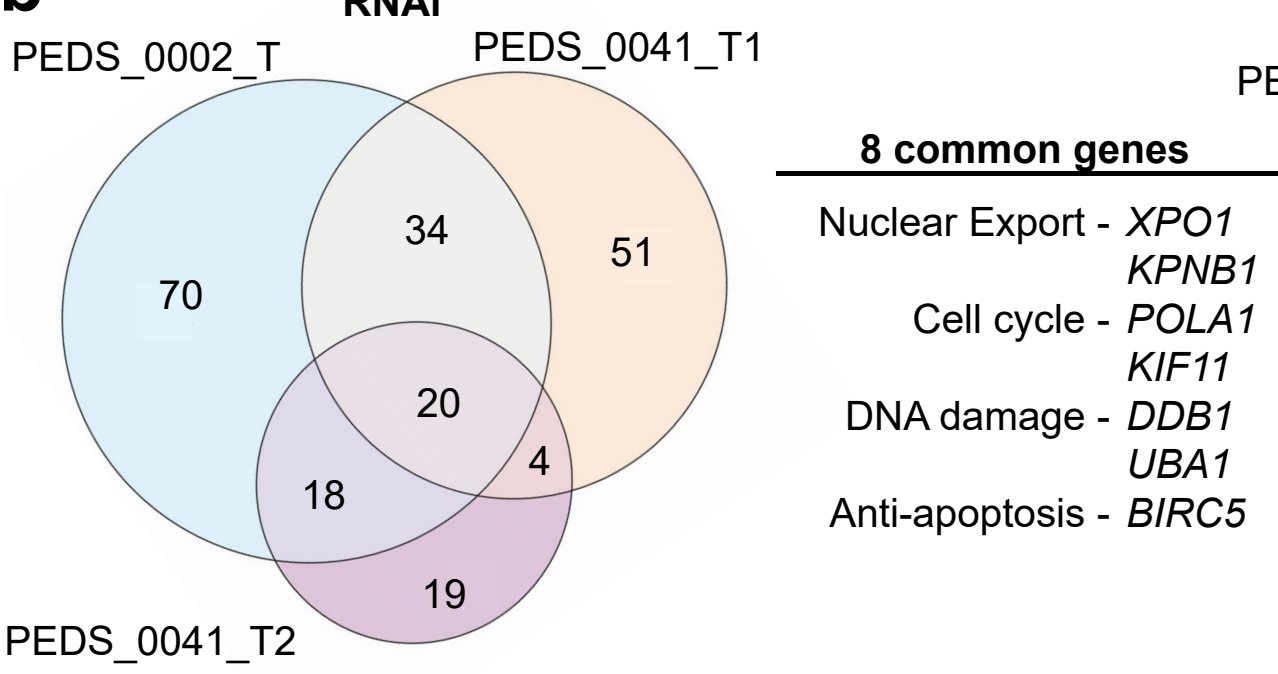

PEDS_0002_T

\section{CRISPR-Cas9}

C

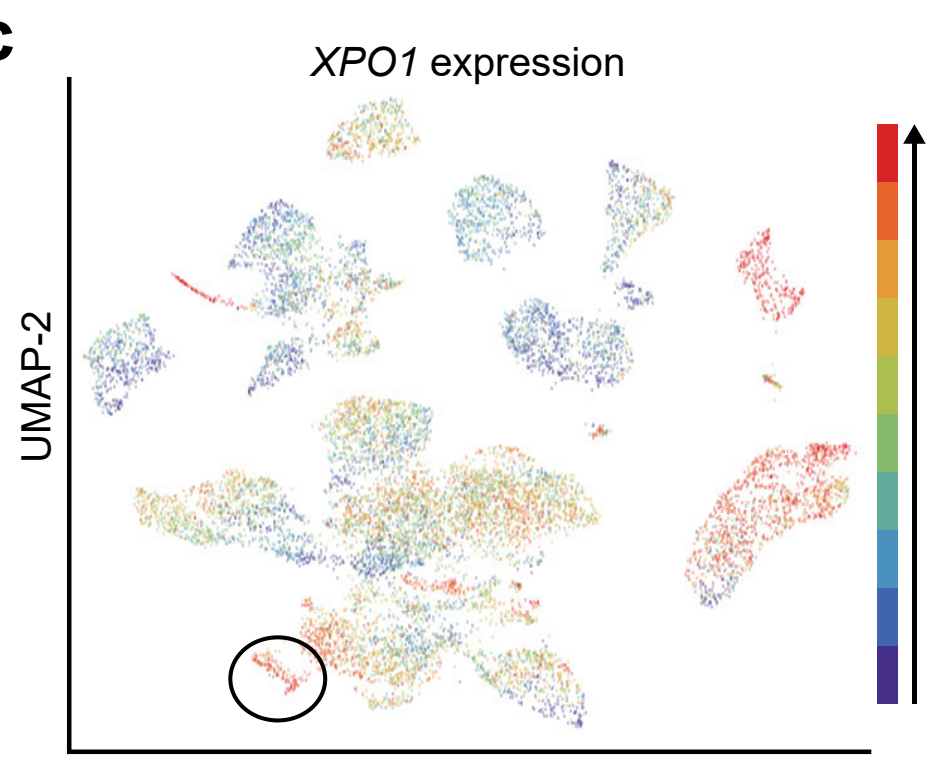

d

$\mathbf{e}$

UMAP-1

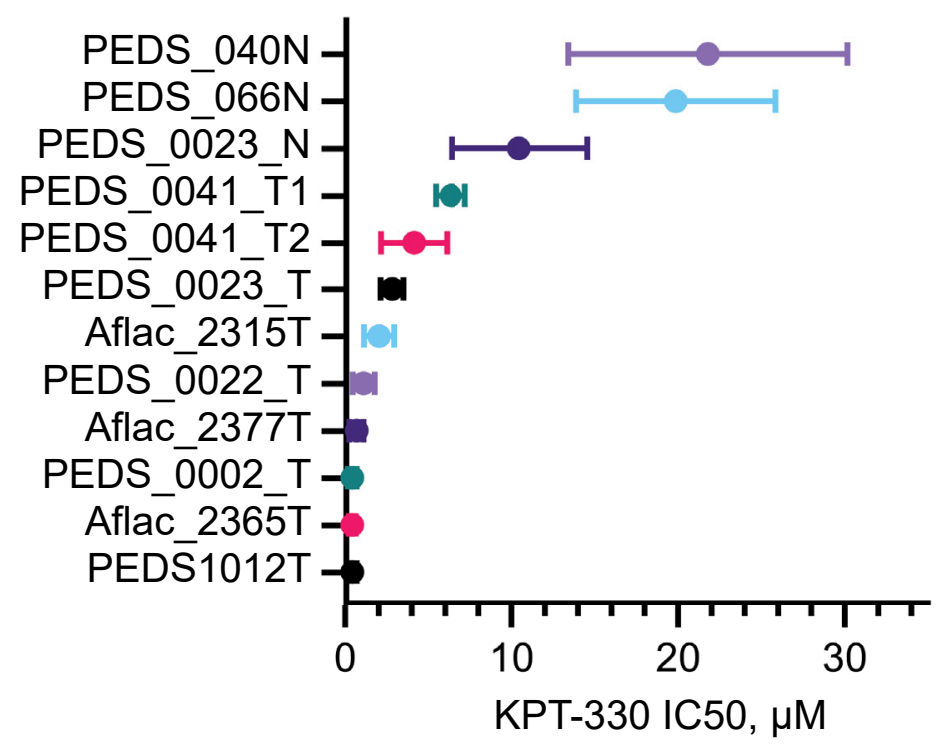

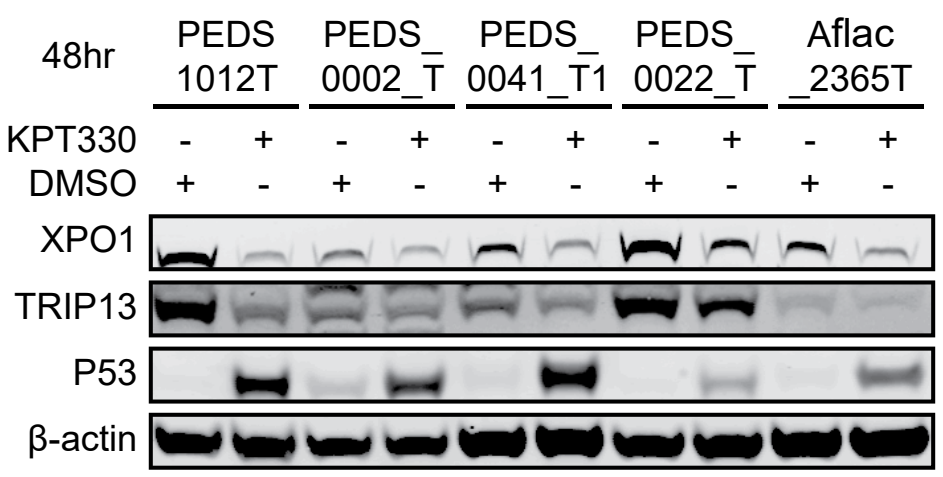


Mittal and Lee et. al.

637 Figure 2: XPO1 is a potential therapeutic target in Wilms tumor cells. (a) Schematic outlining the

638 methodology of CRISPR-Cas9 and RNAi functional screens. (b) RNAi suppression in three cell lines

639 identified 20 common genes which were critical for the survival of WT cells. CRISPR-Cas9 screens

640 identified 24 common genes in three cell lines which were critical for the survival of WT cells. Eight genes

641 overlapped between the RNAi and CRISPR-Cas9 loss-of-function screens. These 8 genes can be

642 categorized under their role in nuclear export, cell cycle, DNA damage and apoptosis. (c) UCSC Treehouse

643 transcriptional data from 12,719 samples showing expression of XPO1 in all tumor types with WT samples

644 circled in black. Blue to red colors signify expression levels with red being the highest among this cohort.

645 (d) Forest plot representing the $\mathrm{IC}_{50}$ of KPT-330 in the panel of WT cell lines and normal cells (ending with

$646 \mathrm{~N})$. Cell viability data is based on biological duplicates with technical triplicates. (e) Immunoblots depicting

647 the decrease in protein levels of XPO1 upon treatment with KPT-330. Data shown are representative of at

648 least biological replicates. 
2 bioRxiv preprint doi: https://doi.org/10.1101/2022.02.23.481521; this version posted February 25, 2022. The copyright holder for this preprint

- G2/M checkpoint genes
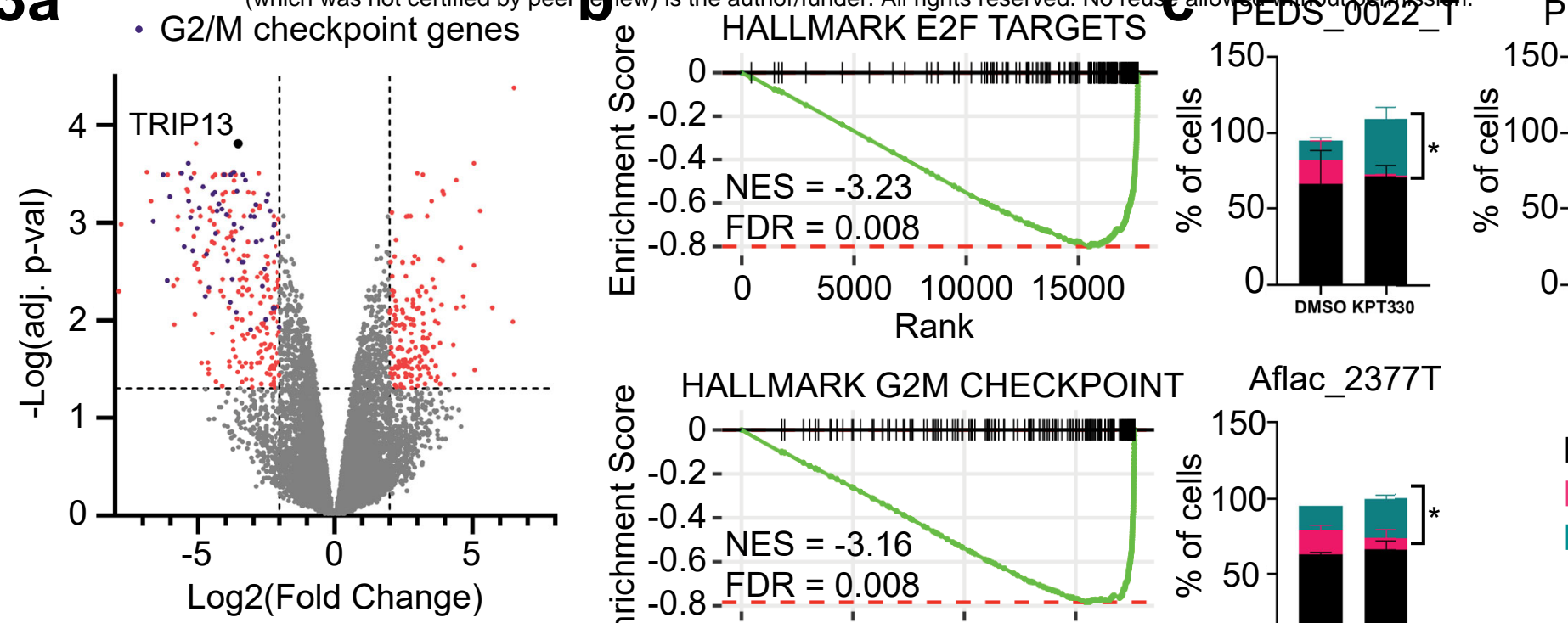

PEDS1012T

d

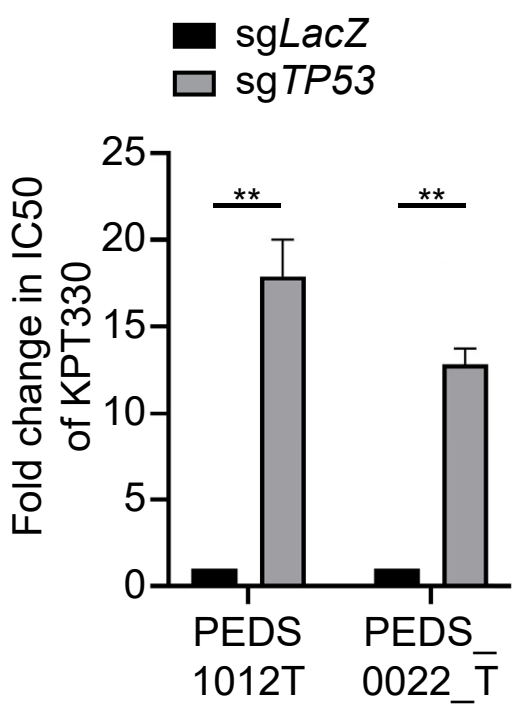

g
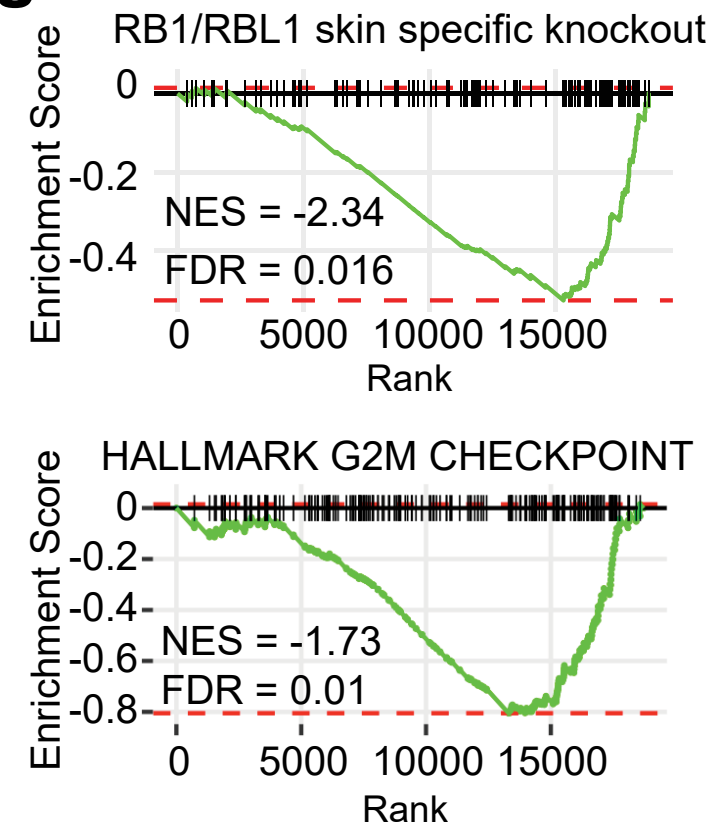
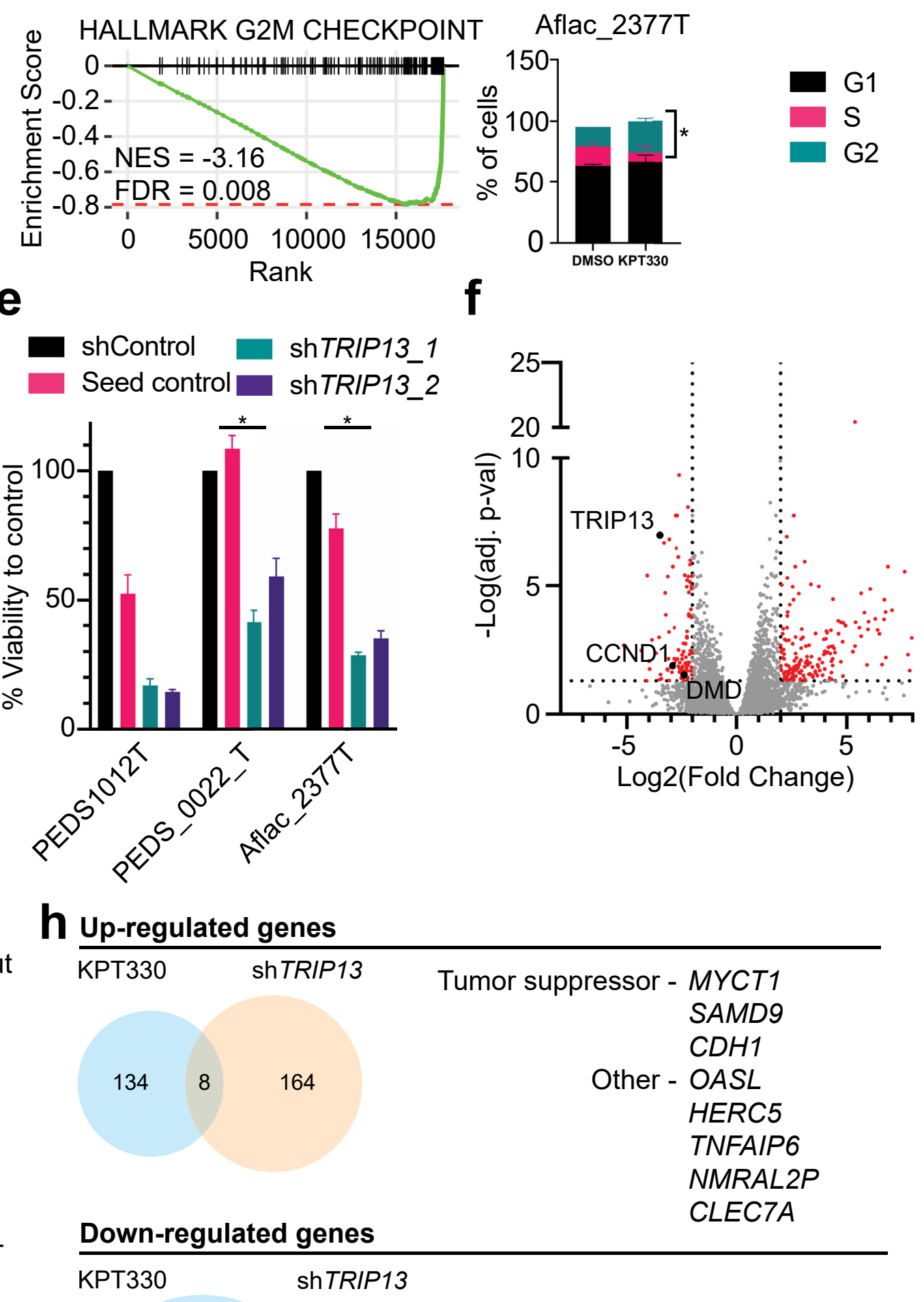

Down-regulated genes

KPT330

shTRIP13

Gene of interest - TRIP13

Cell cycle - ASPM

CDCA7

Other - COLEC12 
Mittal and Lee et. al.

Figure 3: XPO1 inhibition leads to decreased viability through TRIP13 and TP53 axis: (a) Volcano

650 plot representing differential gene expression between the KPT-330 and DMSO treated

651 CCLF_PEDS_0041_T cell line. Scattered points represent genes: the $\mathrm{x}$-axis is the fold change for KPT-330

652 vs DMSO treated CCLF_PEDS_0041_T cells as compared to the p-values. Purple dots represent genes in

653 the Hallmark G2/M Checkpoint gene set. (b) Gene set enrichment analysis (GSEA) enrichment score curves

654 for the E2F and G2M hallmark pathways in the CCLF_PEDS_0041_T cells treated with KPT-330. The

655 green curve denotes the ES (enrichment score) curve, the running sum of the weighted enrichment score in

656 GSEA. (c) KPT-330 and DMSO treated CCLF_PEDS1012T, CCLF_PEDS_0022_T, and AFLAC_2377

657 cells were subjected to cell cycle analysis following flow cytometry. Bar graph representing the proportion

658 of cells across phases of the cell cycle in at least biological replicates and 50k cells counted. (d) Deletion

659 of TP53 significantly increased the $\mathrm{IC}_{50}$ of KPT-330 in CCLF_PEDS1012T and CCLF_PEDS_0022_T.

660 The fold change is based on comparison to a LacZ non-targeting control and based on biological duplicates.

661 Error bars represent mean \pm SD. (e) Change in viability using shTRIP13_1 and shTRIP13_2 across FHWT

662 cell lines as compared to shControl and seed control (to shTRIP13_2). (f) Volcano plot showing the

663 distribution of significant genes up or downregulated following shTRIP13. RNA seq was performed on

664 Aflac_2377T and CCLF_PEDS_0041_T cell lines with shTRIP13 as compared to shControl. Biological

665 replicates performed. TRIP13 is downregulated along with CCND1. (g) Gene set enrichment analysis

666 (GSEA) enrichment score curves for the RB1/RBL1 skin specific knockout and G2M pathways following

667 suppression with shTRIP13. (h) Commonly upregulated and downregulated genes seen in both KPT-330

668 treated or shTRIP13 treated cells. Eight genes were upregulated and four genes were downregulated. 
bioRxiv preprint doi: https://doi.org/10.1101/2022.02.23.481521; this version posted February 25, 2022. The copyright holder for this preprint
(which was not certified by peer $r(\mathbf{D e w}$ ) is the author/funder. All rights reserved. No reuse Gillowed without permission.

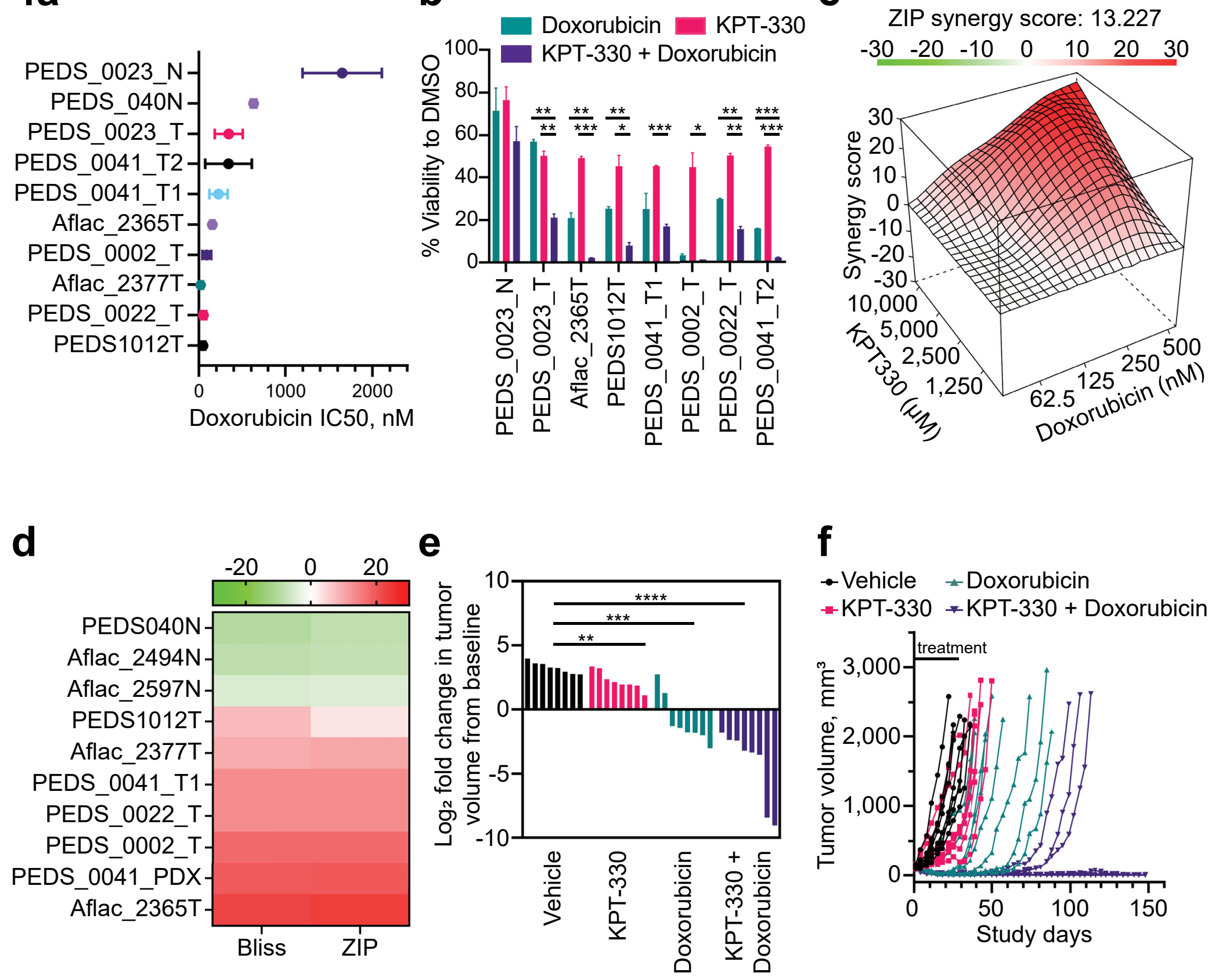

g

- Vehicle -Doxorubicin

-KPT-330 - KPT-330 + Doxorubicin

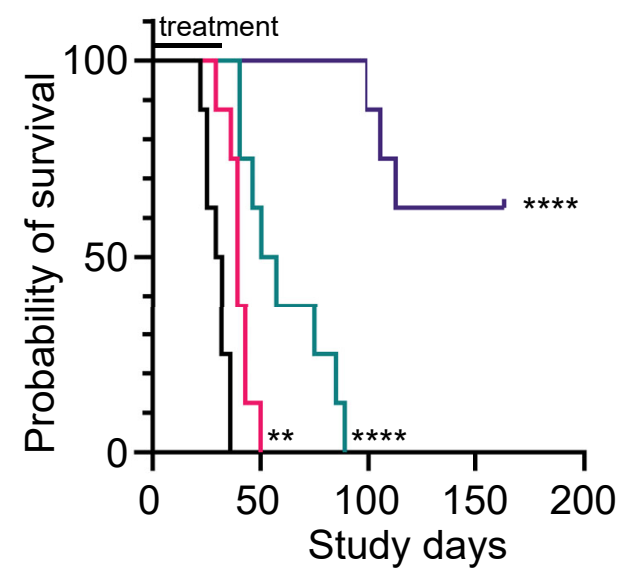


Mittal and Lee et. al.

Figure 4: Combination of doxorubicin and KPT-330 is synergistic in vitro and in vivo (a) IC50s for

670 WT cell lines treated with doxorubicin as compared to normal kidney cells. X-axis is nM. SD shown from

671 at least 2 biological replicates. (b) Bar graph representing the percent viability of WT cells upon treatment

672 with doxorubicin $(100 \mathrm{nM})+\mathrm{KPT}-330(5 \mu \mathrm{M})$ for 72 hours. Error bars represent mean \pm SD from biological

673 triplicates. (c) Representative 3D landscape image of the synergy score for WT cells treated with

674 doxorubicin $(62.5-500 \mathrm{nM})$ and KPT-330 (1.25 - $10 \mu \mathrm{M})$. (d) Heat map showing synergy scores from Bliss

675 and ZIP drug-drug interaction models in WT and normal kidney cells. Range green (antagonistic) to red

676 (additive 0-10 to synergy $>10$ ). (e) CCLF_PEDS_0041 fragments were placed subcutaneously into the hind

677 flank of NSG (NOD-SCID IL2Rgamma null) female mice. The tumor xenografts were treated with vehicle,

678 doxorubicin, KPT-330 or the combination of doxorubicin and KPT-330 for 28 days. Log fold change of

679 tumor volumes were calculated as compared to time of treatment (average $114.3 \mathrm{~mm}^{3}$ ). ** p-value $<0.005$,

$680 * * * \mathrm{p}$-value $<0.0005, * * * * \mathrm{p}$-value $<0.00005$. (f) Line graph depicting the tumor volume across the study

681 days for each mouse in different treatment groups. Treatment lasted 4 weeks from study enrollment and

682 continued until mouse met endpoint or study termination at day 150. (g) Kaplan Meier survival curves

683 representing the probability of overall survival in the patient-derived xenografts ( $\mathrm{n}=8$ per treatment group)

684 treated with the vehicle, KPT-330, doxorubicin and KPT-330 + doxorubucin. ** p-value $<0.005$, **** p-

685 value $<0.00005$. 
bioRxiv preprint doi: https://doi.org/10.1101/2022.02.23.481521; this version posted February 25, 2022. The copyright holder for this preprint (which was not certified by peer review) is the author/funder. All rights reserved. No reuse allowed without permission.

Mittal and Lee et. al.

\section{SUPPLEMENTARY FIGURES AND SUPPLEMENTARY FIGURE LEGENDS}


$\mathbf{S 1}$ bioRxiv preprint doi: https://doi.org/10.1
(which was not certified by pe review) is the author/funder. All rights reserved. Noreuse allowed without permission. CCLF_PEDS_0002_T

CCLF_PEDS_0041_T1

CCLF_PEDS_0041_T2
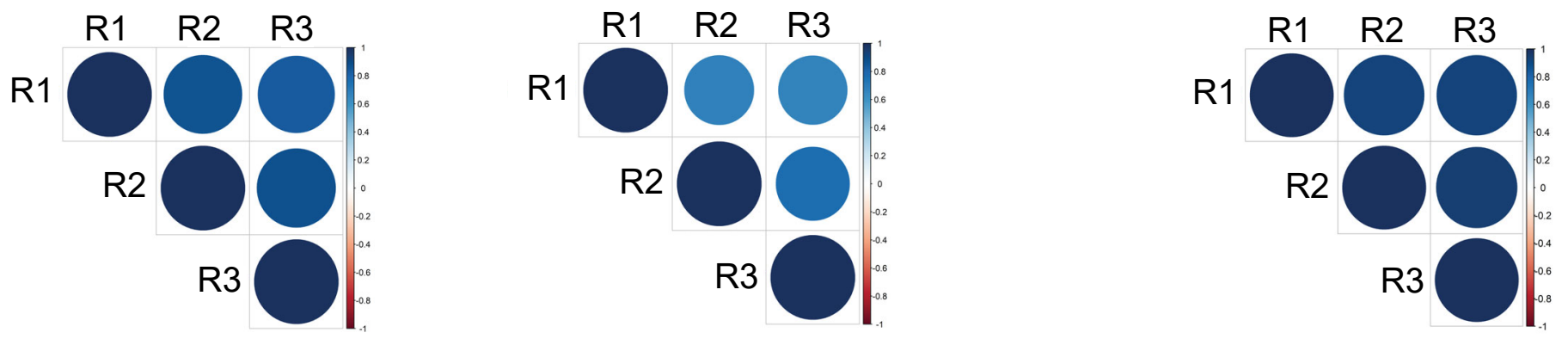

d

CCLF_PEDS_0022_T

e

CCLF_PEDS_0023_T
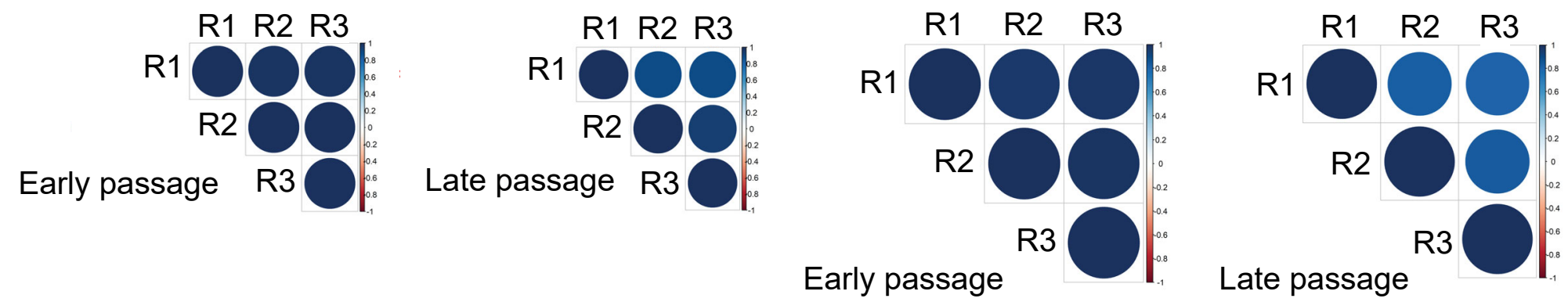

f

CCLF_PEDS_0023_T2

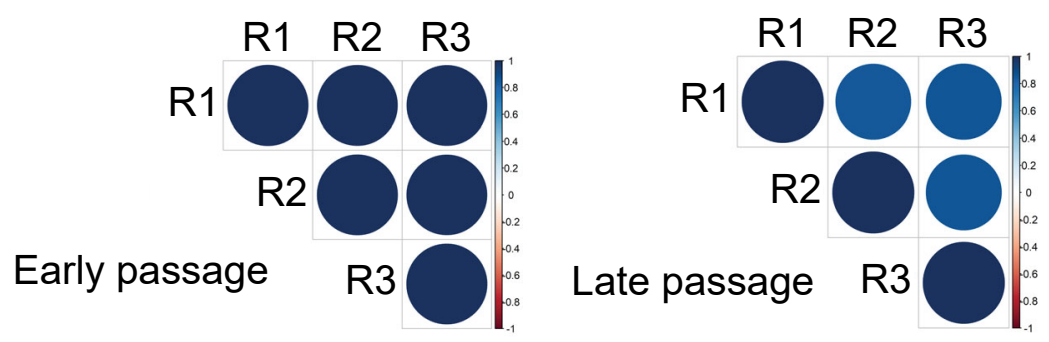


Mittal and Lee et. al.

687 Supp Fig 1: Tight correlation between shRNA and sgRNA loss of function screens among biological

688 replicates. RNAi screens represented in a-c. (a) CCLF_PEDS_0002_T, (b) CCLF_PEDS_0041_T1, (c)

689 CCLF_PEDS_0041_T2. CRISPR-Cas9 screens used early and late passages to determine change in

690 abundance of sgRNAs as shown in d-f. (d) CCLF_PEDS_0022_T, (e) CCLF_PEDS_0023_T, (f)

691 CCLF_PEDS_0023_T2. Right color gradient delineates the Pearson's correlation with anticorrelation as

692 red and correlation as blue. 


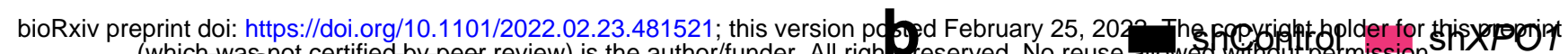
(whighty gnot certifieg $\$$ Elpges review) is the author/funder. All righ

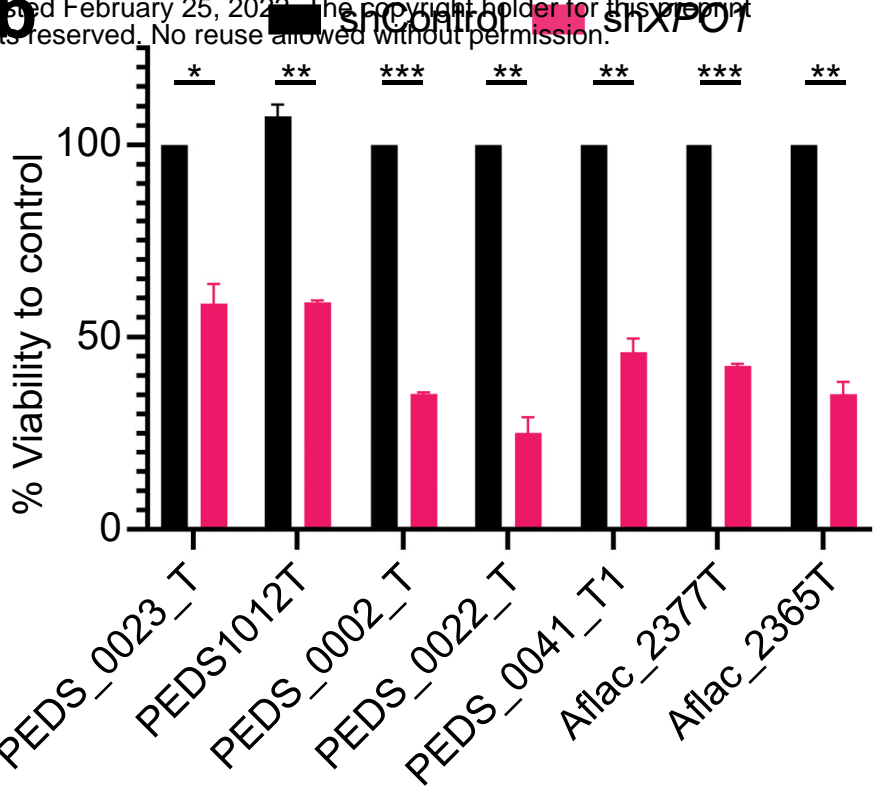

C

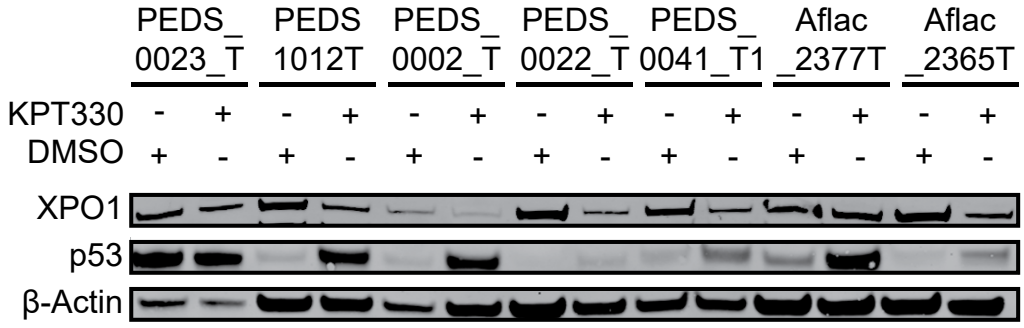

d

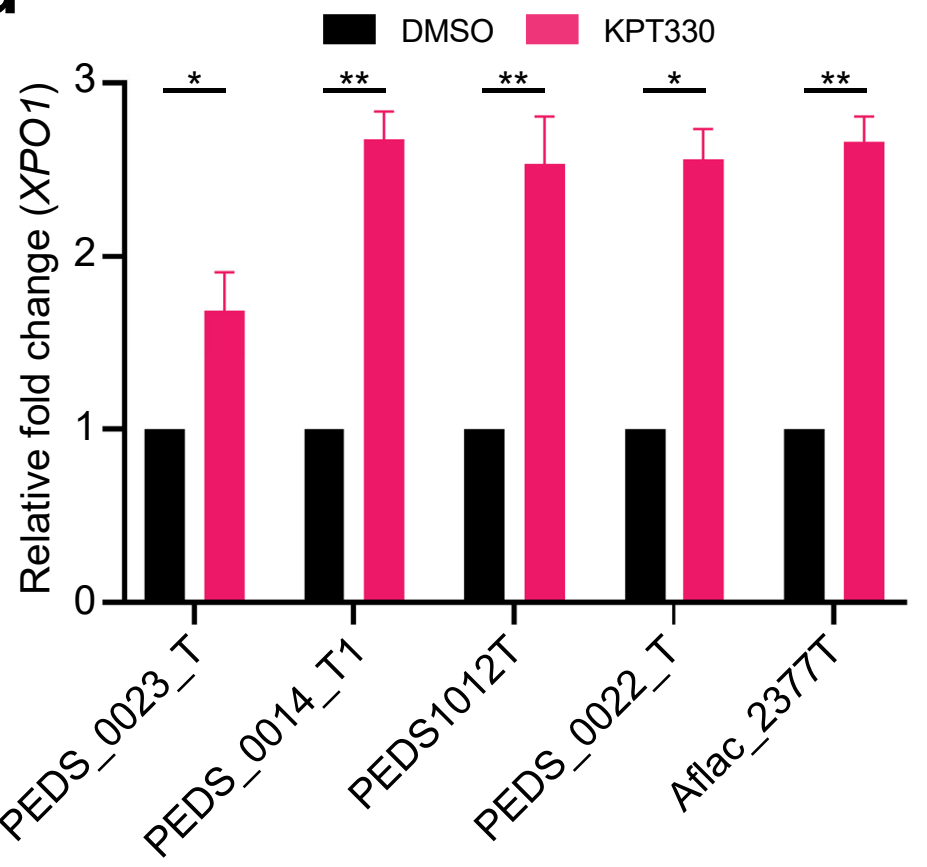


Mittal and Lee et. al.

\section{Supplementary Figure 2: Inhibition of nuclear export in FHWT}

694 (a) Suppression of XPO1 by immunoblot (b) Change in viability using shXPO1 across FHWT cell lines as

695 compared to shControl (c) At 24 hours, XPO1/CRM levels are suppressed with accumulation of TP53

696 across a majority of cell lines. (d) qRT-PCR analysis of XPO1 in KPT-330 and DMSO treated cells. Data

697 was normalized by the amount of TBP, expressed relative to the corresponding value for all the cells and

698 are means \pm SD from at least two biological replicates. $*$ indicates a p-value $<0.05, * *$ p-value $<0.005, * * *$

699 p-value $<0.0005$. 


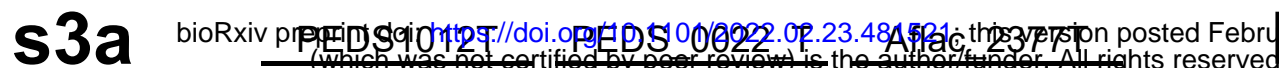

25,2022 . The copyright holder for this preprint

\begin{tabular}{|c|c|c|c|c|c|c|c|c|}
\hline \multirow{2}{*}{ No reuse allow } & \multicolumn{4}{|c|}{ PEDS1012T } & \multicolumn{4}{|c|}{ PEDS_0022_T } \\
\hline & 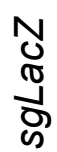 & 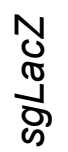 & 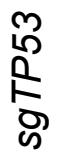 & 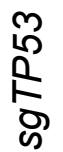 & $\begin{array}{l}\text { N } \\
\text { ত্ } \\
\frac{J}{\sigma} \\
\text { ర্ }\end{array}$ & 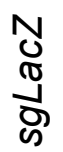 & 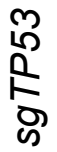 & 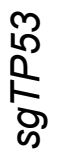 \\
\hline DMSO & + & - & + & - & + & - & + & - \\
\hline КРТ330 & - & + & - & + & - & + & - & + \\
\hline TP53 & & -4 & & & & & & \\
\hline$\beta$-actin & 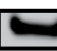 & & & & & & & \\
\hline
\end{tabular}
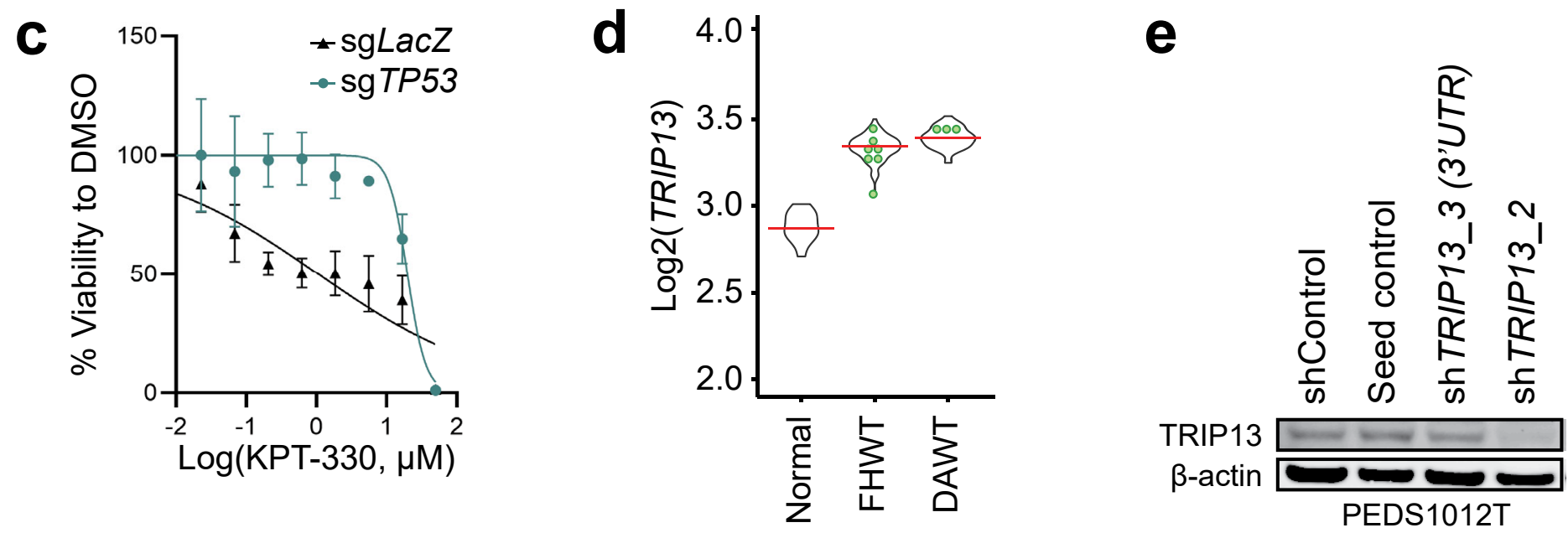

PEDS1012T

$f$
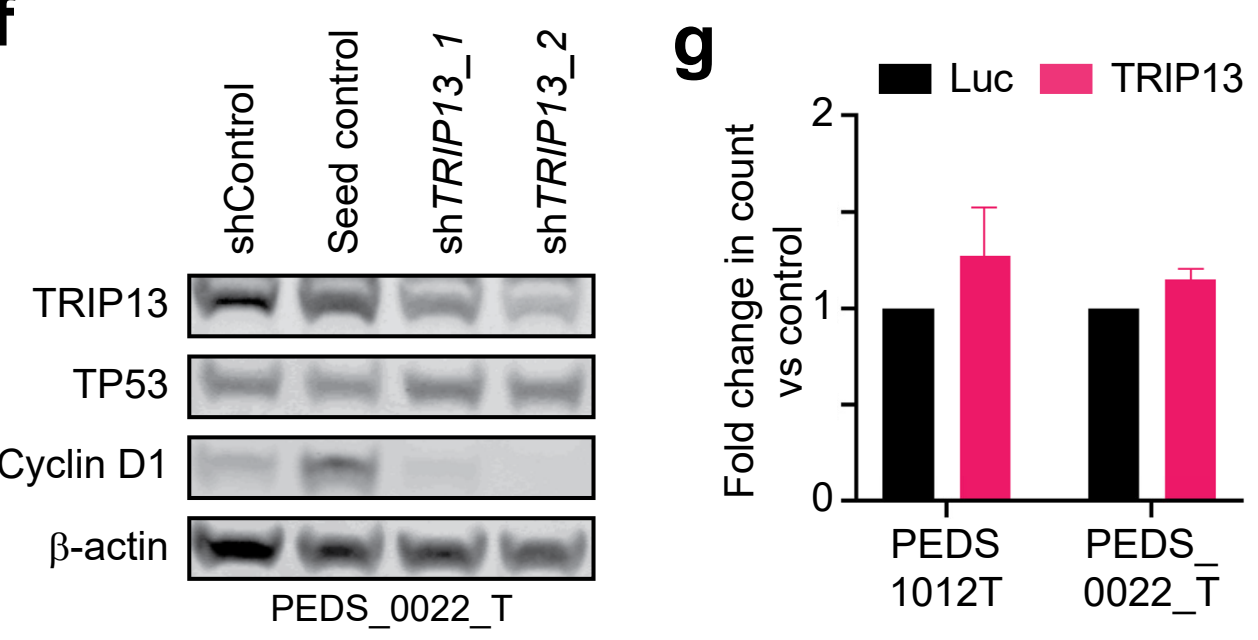

h

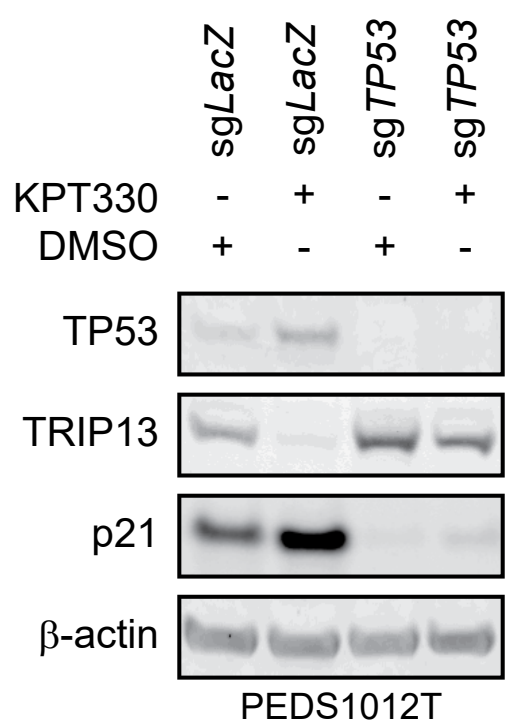


Mittal and Lee et. al.

700 Supplementary Figure 3: Inhibition of nuclear export and TRIP13 in FHWT cell lines. (a)

701 Immunoblots depicting the decrease in protein levels of XPO1 in the cytoplasmic lysates and nuclear

702 accumulation of TP53 in the nuclear lysates upon treatment with KPT-330 at $24 \mathrm{hr}$. (b) We introduced

703 sgRNAs targeting either LacZ or TP53 and confirmed decrease in TP53 following treatment with KPT-

704 330. (c) Dose-response curves for the sgTP53 and $\operatorname{sg} L a c Z$ cells for KPT-330. Error bars represent mean

$705 \pm \mathrm{SD}$ and represent biological replicates. (d) Dot plots representing the expression levels of TRIP13 in

706 Wilms tumor when compared with the normal matched kidney tissue. Immunoblot depicting suppression

707 of TRIP13 in (e) CCLF_PEDS1012T (f) CCLF_PEDS_0022_T (g) Overexpression of TRIP13 leads to

708 modest increase in proliferation as compared to luciferase. (h) TP53 and p21 protein levels increase while

709 TRIP13 levels decrease upon KPT-330 treatment in TP53 wildtype cells. 